\title{
Reference values for areal bone mineral density among a healthy Mexican population
}

\author{
Juan Tamayo, MD, (1) Rodrigo Díaz, PhD, (2) Eduardo Lazcano-Ponce, DrSc, (2) Madeline Muñoz, MSc, ${ }^{(4)}$ \\ Gerardo Huitrón, MD, (3) Elizabeth Halley, MSc, (5) Juan Carlos Díaz-Montiel, MSc, ${ }^{(4)}$ Jyoti Mudgal, PhD, ${ }^{(4)}$ \\ Mauricio Hernández-Ávila, $\mathrm{PhD},{ }^{(2)}$ Jorge Salmerón, $\mathrm{DrSc} .{ }^{(4)}$
}

\begin{abstract}
Tamayo J, Díaz R, Lazcano-Ponce E, Muñoz M, Huitrón G, Halley E, Díaz-Montiel JC, Mudgal J, Hernández-Ávila M, Salmerón J.

Reference values for areal bone mineral density among a healthy Mexican population. Salud Publica Mex 2009;5 I suppl I:S56-S83.
\end{abstract}

Tamayo J, Díaz R, Lazcano-Ponce E, Muñoz M, Huitrón G, Halley E, Díaz-Montiel JC, Mudgal J, Hernández-Ávila M, Salmerón J.

Valores de referencia para la densidad mineral ósea expresada en área para una población mexicana sana. Salud Publica Mex 2009;5 I supl I:S56-S83.

\section{Resumen}

Objetivo. Comparar la influencia de la etnicidad en la prevalencia de osteopenia y osteoporosis en varias poblaciones mexicanas utilizando dos bases de referencia normal de densitometría de rayos $X(D X A)$ : referencia del fabricante que incorpora hispanos en Estados Unidos y datos de una población mestiza mexicana. Material y métodos. Un total de 9946 sujetos de población mestiza mexicana participantes en una cohorte de largo plazo dirigida al estudio de estilos de vida y ocurrencia de enfermedades crónicas; de los cuales 6487 sujetos sanos de ambos sexos, con edad entre los 7 y los 80 años, fueron utilizados para determinar los valores $\mathrm{T}, \mathrm{Z}$, de acuerdo a los criterios de la OMS, así como a los valores de masa ósea pico. Se compararon los casos de densidad mineral ósea anormal de acuerdo a los valores de referencia del fabricante y los valores de masa ósea pico de la población mestiza. Resultados y conclusión. Las bases de referencia del fabricante subestima significativamente el número de casos con densidad mineral ósea anormal en la población mestiza mexicana.

Palabras clave: densitometría ósea, valores normales referencia, etnicidad; México
Key words: bone densitometry, normal reference values, ethnicity, Mexico

(I) Comité Mexicano para la prevención de la Osteoporosis.

(2) Centro de Investigación en Salud Poblacional, Instituto Nacional de Salud Pública.

(3) Centro de Investigación en Ciencias Médicas, Universidad Autónoma del Estado de México.

(4) Unidad de Investigación Epidemiológica y en Servicios de Salud, Morelos. Instituto Mexicano del Seguro Social.

(5) Unidad de Enseñanza, Investigación y Calidad. Instituto de Salud del Estado de México.

Received on: October 10, 2008 - Accepted on: December II, 2008

Address reprint requests to: Dr. Eduardo Lazcano-Ponce, INSP, Centro de Investigación en Salud Poblacional. Av. Universidad Nº 655 Col. Santa María Ahuacatitlán 62508, Cuernavaca, Morelos, Mexico.

E- mail: elazcano@insp.mx 
$\mathrm{O}$ steoporosis and its most common complication, fragility fractures, are well recognized internationally as public health problems. ${ }^{1-6}$ Mexico is no exception, as femoral fractures account for nearly 40000 hip replacement surgeries performed in the nation each year ${ }^{7-13}$ with an estimated direct cost of 800000 US dollars. In 1994, the World Health Organization (WHO) developed criteria to classify bone mineral density (BMD) values as an estimation of lifelong relative fracture risk ${ }^{14}$ and to classify people as osteopenic or osteoporotic based on the low energy dual x-ray absorptiometry (DXA) T-score values of healthy young adults (20 to 40 years old). BMD is influenced by genetic and lifestyle factors which may significantly vary between ethnic groups. BMD reference values for the US Hispanic population are available from the National Health and Nutrition Examination Survey (NAHNES) and can be found in clinical equipment software. However, there are no such reference values available for the healthy Mexican population, which is ethnically distinct from the US Hispanic population.

Over the last 15 years, DXA has been widely used to determine bone mineral density (BMD) at different anatomical sites. This technology has been used for early detection of individuals at high risk of osteoporotic fractures. According to these WHO criteria, T-scores should be developed based on mean BMD values + / - standard deviation (SD) among a reference population of healthy young adults (20 to 40 years old). These criteria have been adopted as the optimal bone strength reference values to qualify as osteopenic or osteoporotic, based on the BMD evaluation. A T-score value above 1.0 SD is considered normal, between -1.0 and -2.5 SD as osteopenia, and below -2.5 as osteoporosis. ${ }^{14-16} \mathrm{~A}$ Z-score was also developed as a comparison for BMD determinations against age- and gender-matched healthy individuals. The lifelong relative fracture risk for the osteopenic range increased to 1.6 and it was over 2.0 for the osteoporotic range. The prediction is stronger if data is analyzed for the specific region of interest, namely, if one measures femoral neck results to predict the relative risk of having a femoral neck fracture; in such an example the relative risk increases to over 2 for an osteopenic BMD value and to over 6 for osteoporotic values. $^{14}$

In order to minimize the differences between reference populations, researchers sought to generate BMD reference values for different ethnic groups in the United States based on National Health and Nutrition Examination Survey (NHANES) data. ${ }^{17-19}$ T-scores for Hologic and Lunar instruments have thus been developed for specific anatomical regions among 20 to 40 year-old healthy adult men and women and for different ethnici- ties. During the last 10 years, these estimates have been used as reference values for operational and diagnostic purposes at the clinical level.

In recent years, the use of BMD T-score values for different ethnic groups and countries has been questioned in light of increasing evidence that the true maximal bone strength at skeletal maturity is the peak bone mass (PBM), representing the optimal bone mass of an individual. ${ }^{20} \mathrm{It}$ is now believed that a T-score computed using this optimal bone strength value (PBM) may be a better reference than the mean of a healthy young adult population because bone mass is still increasing ${ }^{21-22}$ in individuals between 20 and 30 years of age. Therefore, including sub-optimal BMD estimates in the calculation of T-scores may lead to a significant underestimation of osteopenic and/or osteoporotic cases.

BMD is the strongest predictive factor of fractures that can be assessed with high reproducibility and specificity. ${ }^{22-26}$ To use this predictor in Mexico, it is thus crucial to have reference PBM values for the Mexican population in order to compute a T-score that truly reflects optimal bone strength at skeletal maturity. Adopting national standards based on BMD reference values and T-score estimates for our population may improve the assessment of osteoporosis in Mexico. Since anti-fracture interventions provide over 50 percent protection against osteoporotic fractures after the first year of treatment, improved ability to assess fracture risk would improve both public health decision making and clinical outcomes. ${ }^{3}$

The aim of the study is to provide BMD reference values based on a healthy, urban Mexican population aged 7 to 80 years and stratified by sex, enabling us to estimate the PBM reference values for the Mexican population. These values are necessary for computing a T-score that truly reflects optimal bone strength at skeletal maturity in this population. Using these BMD reference values, we are able to calculate optimal BMD $\mathrm{T}$ - and Z-scores, both according to WHO criteria and PBM values. Adopting these reference values as a national standard can improve public health practices and clinical assessment of osteoporosis in Mexico.

\section{Material and Methods}

Study Population: The present analysis was performed with healthy employees and their healthy relatives from three different health and academic institutions: Instituto Mexicano del Seguro Social (IMSS) and the Instituto Nacional de Salud Pública (INSP) in Cuernavaca, Morelos, Mexico, and employees from the Universidad Autónoma del Estado de México (UAEM) in Toluca, State of Mexico. Subjects recruited for the study were 
participating in the first stage of an ongoing, long-term cohort study focusing on lifestyle and health, approved by the respective ethical committees of each participating institution; informed consent was obtained in all cases. Out of a total population of 13275 study candidates identified between March 2004 and April 2006, 9467 employees were invited to participate in the cohort study and a total of 8307 adults were formally enrolled. Those participants who were parents of children 7 to 18 years of age (about $20 \%$ of the employees) were also asked to invite their children to be part of the study. Of those willing to participate, 1639 youths were formally invited and enrolled in the study. Thus, a total of 9946 subjects between 7 and 92 years of age were formally recruited. BMD determinations in this study population constitute part of a more extensive epidemiologic evaluation designed to prospectively evaluate different hypotheses about the relationships between lifestyle and chronic disease occurrence in Mexico.

For the present analysis, we excluded subjects older than 80 years of age as well as participants with chronic diseases that may affect bone metabolism and/or alter the BMD values, such as: diabetes $(n=631)$, cancer $(n=96)$, obesity ( $\mathrm{BMI} \geq 35, \mathrm{n}=262)$, arthritis $(\mathrm{n}=286)$, kidney stones $(\mathrm{n}=182)$, renal dysfunction (creatinine $>2 \mathrm{mg} / \mathrm{dL}$ for men, $>1.8 \mathrm{mg} / \mathrm{dL}$ for females, $\mathrm{n}=283$ ) previously diagnosed osteopenia/osteoporosis or fractures after the age of 45 $(\mathrm{n}=64)$, use of medications that may alter bone metabolism $(n=69)$, and weight change in previous year $(\geq 10 \mathrm{~kg}, \mathrm{n}=89)$. We also excluded pregnant women and those women who were unable to undergo BMD measurements. The final healthy reference population for the present analysis is 6478 . This large sample allowed us to estimate PBM as well as T- and Z-scores. The general characteristics of this population are depicted in table I.

\section{BMD assessment}

Bone density measurements were performed at the nondominant proximal femur, the lumbar spine (L1-L4), and whole body using a DXA Lunar DPX NT instrument. The user manual instructions and International Society of Clinical Densitometry (ISCD) procedures were strictly followed. ${ }^{16,27}$ Standardized densitometry technicians performed all BMD measurements. Standard calibration of instruments was performed daily using the phantom provided by the manufacturer; technicians ensured that the daily variation coefficient (VC) was within normal operational standards and in vivo $\mathrm{VC}$ was lower than $1.5 \%$. Results obtained by DXA are expressed as grams of hydroxyapatite per square centimeter (WHO Technical Report Series: Prevention and Management of Osteoporosis. http: / / whqlibdoc.who. int/trs/WHO_TRS_921.pdf).

\section{T- and Z-scores estimates procedures}

In order to compute T-scores by specific site (total body, femur and lumbar spine), we first estimated the reference value for PBM in our population following a previously reported procedure. ${ }^{28}$ Briefly, we calculated the difference in $\mathrm{g} / \mathrm{cm}^{2}$ between the BMD of a subject and the PBM for the corresponding sex. The difference between these values is expressed as standard deviation (SD), or in other words, the difference between the studied subject and the PBM is standardized. The Tscore obtained with this method represents the difference expressed by the number of standard deviations between the Young Normal (YN) value of the population of the same sex and the BMD of the subject studied. This sequence is summarized by the following formula:

Table I

Population characteristics for a cohort study on lifestyle AND health. Mexico

\begin{tabular}{|c|c|c|c|c|c|c|c|c|c|c|c|c|}
\hline \multirow[b]{4}{*}{ Age } & \multicolumn{6}{|c|}{ Men $=1849$} & \multicolumn{6}{|c|}{ Women $=4629$} \\
\hline & \multicolumn{2}{|c|}{$\begin{array}{c}7-18 \text { years old } \\
n=483\end{array}$} & \multicolumn{2}{|c|}{$\begin{array}{c}19-50 \text { years old } \\
n=1004\end{array}$} & \multicolumn{2}{|c|}{$\begin{array}{c}51 \text { and }+ \\
n=362\end{array}$} & \multicolumn{2}{|c|}{$\begin{array}{c}7-18 \text { years old } \\
n=568\end{array}$} & \multicolumn{2}{|c|}{$\begin{array}{c}19-50 \text { years old } \\
n=3100\end{array}$} & \multicolumn{2}{|c|}{$\begin{array}{c}51 \text { and }+ \\
n=961\end{array}$} \\
\hline & Mean & SD & Mean & SD & Mean & $S D$ & Mean & SD & Mean & SD & Mean & SD \\
\hline & 13.03 & 3.10 & 36.50 & 8.84 & 60.99 & 8.24 & 13.44 & 3.12 & 36.28 & 8.78 & 60.87 & 8.21 \\
\hline Height & 156.06 & 17.01 & 169.27 & 6.72 & 166.53 & 6.60 & 151.88 & 11.82 & 156.90 & 28.21 & 153.05 & 5.85 \\
\hline BMI & 20.56 & 4.58 & 26.37 & 3.43 & 26.50 & 3.15 & 21.05 & 4.21 & 25.44 & 3.75 & 26.93 & 3.51 \\
\hline $\begin{array}{l}\text { Abdominal cir- } \\
\text { cunference }\end{array}$ & 76.07 & 13.28 & 91.11 & 9.03 & 94.47 & 8.57 & 77.00 & 11.71 & 85.64 & 10.00 & 94.68 & 10.99 \\
\hline$\%$ fat & 24.18 & 10.97 & 28.41 & 6.71 & 29.87 & 6.32 & 35.23 & 9.01 & $4 I .11$ & 6.48 & 43.94 & 5.76 \\
\hline
\end{tabular}




$$
T=\frac{(B M D-Y N)}{S D}
$$

Similarly the Z-value or Z-score represents the comparison between the subject's BMD and the mean BMD value of the healthy adult population of the same age and sex, referred to as Adult Mean Normal (AMN). It is also used as a measure of the standard deviation of the reference population. The formula is:

$$
Z=\frac{(B M D-A M N)}{S D}
$$

\section{Results}

We evaluated 6478 healthy men and women from 7 to 80 years of age who did not have conditions affecting bone health, out of a total of 8307 adults and 1603 of their children formally enrolled in the first stage of an ongoing, long-term cohort study focusing on lifestyle and health. This recruitment strategy allowed us to have a cohort with healthy bones resembling the real ethnical mixture of a socioeconomic urban middle class population reflecting a wide sector of the Mexican population.

As shown in figure 1, BMD increases from infancy on and reaches PBM sometime between the late twenties and the mid thirties. Once PBM is reached, there is a progressive decrease in BMD with advancing age; these values curve as the population grows older and vary by sex and by skeletal region. Male BMD values are always greater than female BMD values. As shown in Figure 1, males reach the PBM subtotal of the skeleton and whole body BMD values (excluding the skull) between ages 22 and 25, while females do so at 27. Males reach PBM of the total femur measurement at age 23, while females reach it at age 27. Males and females both reach PBM of the lumbar spine at age 32 .

Z-score values are depicted in figure 2. The curves for female BMD values are dramatically different than those for male values; male values are greater than female values starting in early childhood and continuing throughout life. It should also be noted that the values decrease less dramatically for males in later adulthood compared to females. Z-scores reflect a comparison of the individual patient compared to age- and sexmatched controls and can be used to evaluate follow-up studies in other clinical applications.

According to WHO recommendations, computation of the T-score is necessary to establish the cut-off
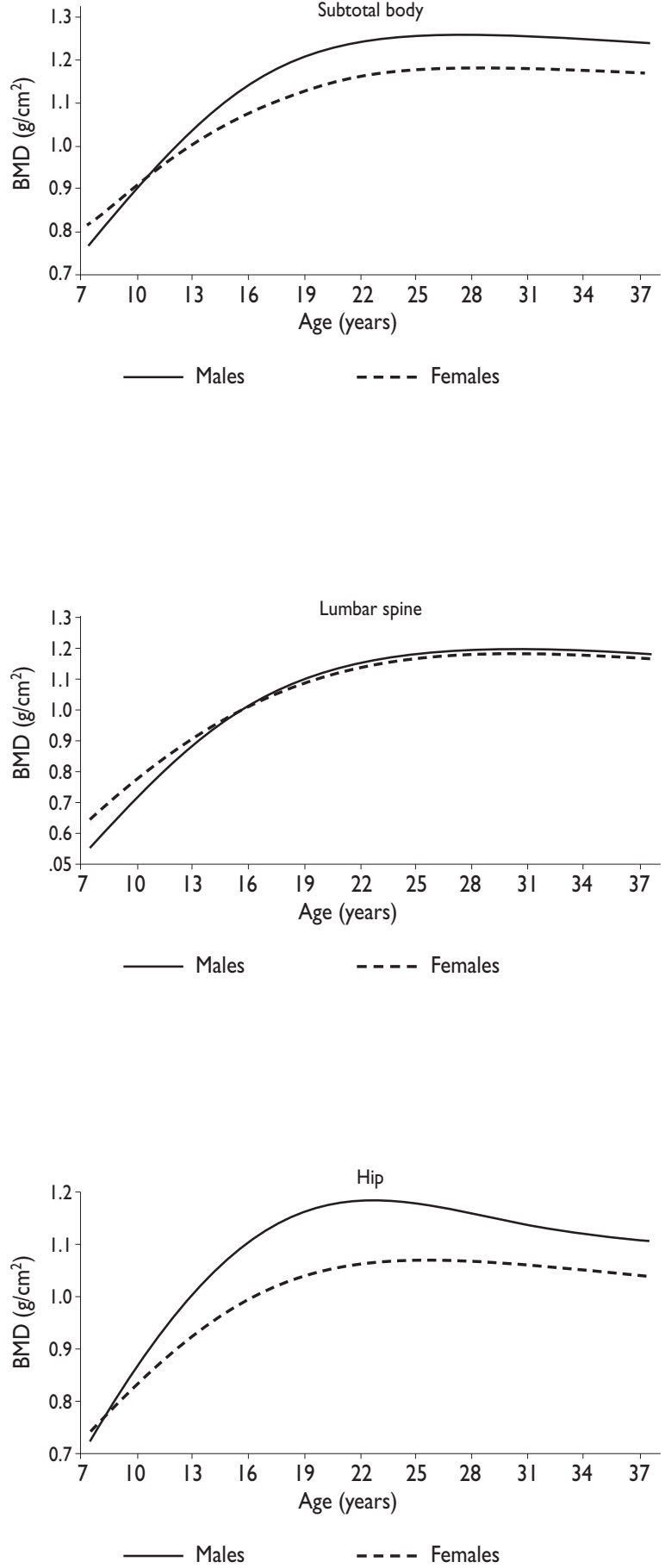

Figure I. BONE MINERAL DENSITY DISTRIBUTION FOR THREE anATOMICAL REGIONS IN MeXican Males aND females from 6 TO 27 YEARS OLD 

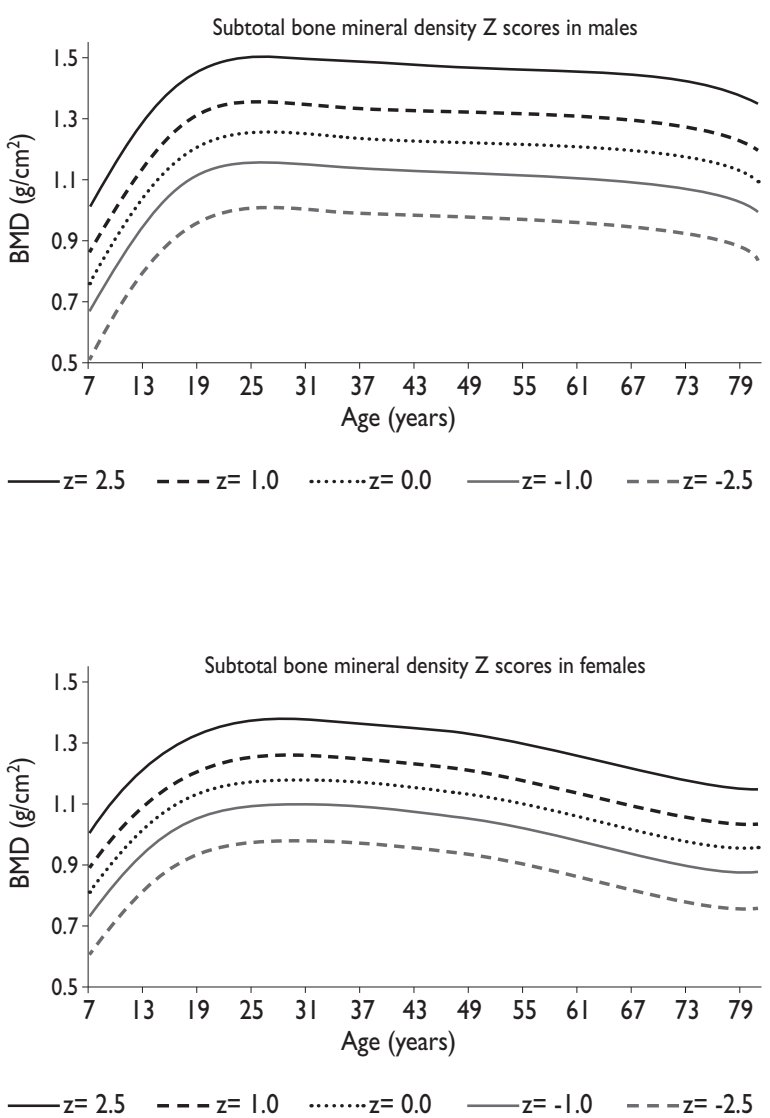

Figure 2. Reference parameters for bone mineral denSity among a Mexican population Classified by aGe AND SEX

point for defining the presence of osteopenia and/or osteoporosis in each specific population. Therefore, both the method for computing T-scores and the reference population used for these computations must be optimized to generate accurate frequency estimates of these health problems. Currently, using instruments on one population that have been calibrated for another can thus impede accurate measurement. The DEXA instruments used in Mexico compute T-scores according to the BMD mean derived from a reference population of healthy US Hispanics between ages 20 and 40 . In table II, we document the differences in the number of osteopenic and osteoporosis cases that occur when US Hispanics versus Mexicans are used as reference populations.
Our results indicate that, by using the normal reference T-scores obtained from the BMD values for the normal female population aged 20 to 40 in our cohort to classify the individual BMD values obtained from two large patient populations (the abnormal population in our cohort and the BMD data obtained and kindly provided to us by Deleze, Cons et al. [previously used for another publication $\left.{ }^{29}\right]$ ) that included 4460 women aged 50 and older who were classified as normal, osteopenic or osteoporotic by 10 densitometry centers located across the country, a higher prevalence of osteopenia and osteoporosis is observed compared to the prevalence obtained using the reference population of healthy US Hispanics integrated in the densitometer by the manufacturer (see figure 3 and tables A.1-A.4 at the appendix). If we restrict the reference population to only those between the ages of 30 to 40 years, there is not a greater underestimation of cases of osteopenia and osteoporosis found using the manufacturer's database (figure 3). If the T-score is computed using the PBM value instead of mean BMD, which we consider to be a more realistic way to define the optimal bone quality reached at skeletal maturation, ${ }^{21}$ the degree of discrepancies between the manufacturer's and our estimates of osteopenia and osteoporosis are substantially similar using the T-scores obtained from either of our Mexican populations (women ages 20 to 40 or ages 30 to 40 years old) (figure 3).

It is clear that by using the manufacturer's T-score values to classify our abnormal Mexican population, we are underestimating the number of abnormal BMD cases. Using either our own T-scores calculated for populations aged 20 to 40 or 30 to 40 , or our PBM, we found that $24.22 \%$ of the cohort population is osteopenic, whereas using the manufacturer's T-score for this value is only $16.52 \%$. The results are similar for osteporotic classification: using PBM we detect a prevalence of $9.73 \%$ versus $6.79 \%$ when using the manufacturer's values. Analyzing the data obtained from the Deleze, Cons cohort with the same criteria, it also became clear that there is a significant underestimation, restricted only to the femoral neck region (tables A.1-A.4 at the appendix).

Tables III.1-III.2 provide a comprehensive reference for the PBM-derived T-score values for a male and female Mexican population. These tables include reference values for both sexes for the 1) total skeleton (excluding the head from whole body scans), 2) the complete proximal femur, and 3) the lumbar region (L1-L4). Tables IV.1-IV.18 describe the Z-score for the same population, depicting each age and the corresponding BMD. These 
Table II

CONCORDANCE OF OSTEOPENIA AND OSTEOPOROSIS PREVALENCE ACCORDING TO DIFFERENT T SCORE COMPUTATIONS DERIVED FROM DIFFERENT REFERENCE POPULATIONS

Classified according to manufacturer's*

Classified according to manufacturer's*

\begin{tabular}{cccc} 
Classified according to BMD mean of a & $20-40$ years old Mexican population \\
\hline Normal & Osteopenia & Osteoporosis & Total \\
$\%$ & $\%$ & $\%$ & $\%$
\end{tabular}

\begin{tabular}{lrrrr} 
Normal (\%) & 68.57 & 8.13 & 0.00 & 76.70 \\
\hline Osteopenia(\%) & 0.04 & 14.29 & 2.18 & 16.52 \\
\hline Osteoporosis(\%) & 0.00 & 0.45 & 6.34 & 6.79 \\
\hline Total (\%) & 68.61 & 22.87 & 8.52 & 100.00
\end{tabular}

Classified according to BMD mean of a 30-40 years old Mexican population

\begin{tabular}{rrrr}
\hline Normal & Osteopenia & Osteoporosis & Normal \\
$\%$ & $\%$ & $\%$ & $\%$
\end{tabular}

\begin{tabular}{lrrrr} 
Normal (\%) & 66.92 & 9.77 & 0.00 & 76.70 \\
\hline Osteopenia(\%) & 0.03 & 13.67 & 2.82 & 16.52 \\
\hline Osteoporosis(\%) & 0.00 & 0.48 & 6.31 & 6.79 \\
\hline Total (\%) & 66.95 & 23.92 & 9.13 & 100.00
\end{tabular}

Classified according to Peak Bone Mass estimate in Mexican population

Normal Osteopenia Osteoporosis Normal

$\% \quad \% \quad \%$

Classified according to manufacturer's*

\begin{tabular}{lrrrr} 
Normal (\%) & 66.02 & 10.68 & 0.00 & 76.70 \\
\hline Osteopenia(\%) & 0.03 & 13.37 & 3.12 & 16.52 \\
\hline Osteoporosis(\%) & 0.00 & 0.17 & 6.61 & 6.79 \\
\hline Total (\%) & 66.05 & 24.22 & 9.73 & 100.00
\end{tabular}

*T score from the total body BMD mean derived from a 20 - 40 year old, US Hispanic population

tables can be used manually to find the T-score and Zscore for the urban Mexican population.

\section{Discussion}

WHO guidelines are helpful for clinically adapting previous definitions of osteoporosis; practically speaking, these guidelines allow for greater accuracy when describing the extent and characteristics of osteoporosis. However, the expert panel that created these guidelines recognized that they would likely change as new knowledge was gathered..$^{30}$ Today, it has become quite evident that fracture risk is also affected by factors other than BMD; not all individuals with low BMD will fracture, while some with normal BMD will. ${ }^{31}$ Also, WHO criteria define low BMD without regard to an individual's age, missing a key element of fracture risk. For example, though a 40 year-old and 65 year-old woman may have the same BMD levels, the older woman's probability of suffering a fracture is increased by the concurrent presence of other risk factors. ${ }^{33-35}$ In addition to excluding age from risk assessment, $\mathrm{WHO}$ criteria do not use PBM values as the optimal bone quality, using the less accurate mean BMD distribution for young adults (20 to 40 years old). The rationale behind the use of this age range is not clearly identified, nor is it clear how to form a reference group for populations that include different racial or ethnic groups. .2-26, $32^{2}$ 


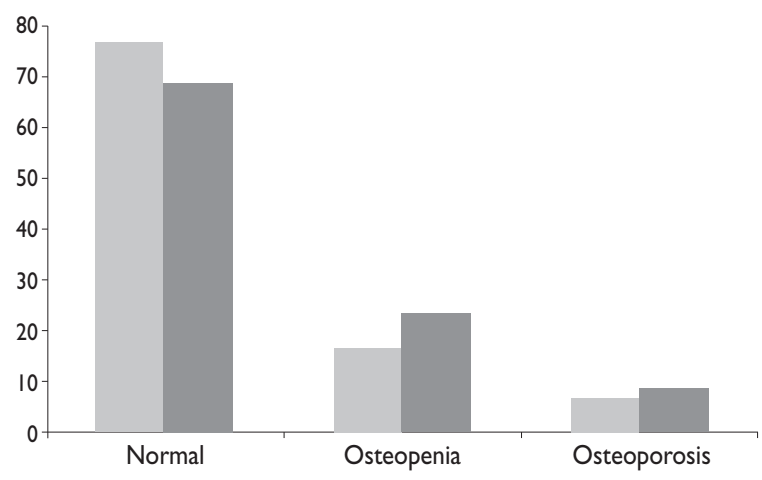

Classified according to manufacturer

Classified according to mean BMD for a Mexican population ages $20-40$ years old

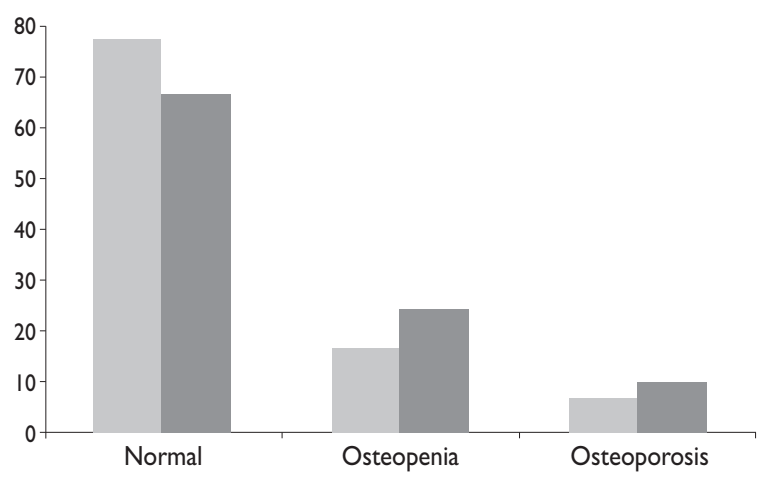

Classified according to manufacturer

Classified according to peak bone mass estimate

for the Mexican population

Figure 3. Concordance of osteopenia AND Osteoporosis PREVALENCE ACCORDING TO DIFFERENT T-SCORE COMPUTATIONS DERIVED FROM DIFFERENT REFERENCE POPULATION

For Mexico, as well as for many other developing countries, the importance of having BMD T-score reference values that reflect the population's unique characteristics has become a critical issue. ${ }^{33,34}$ Several studies have shown that the reference data provided by manufacturers for a US Hispanic population may lead to dangerous underestimations of the population at risk. ${ }^{18,34-38}$ The reference databases provided by manufacturers do not reflect the complex heritage of the Mexican population, also called mestizo, which reflects both indigenous Mexican and Spanish ancestry.
To address a few of these emerging issues, we used the worker's cohort study data with the T-score reference values that reflect the population's unique characteristics to study bone density data from a large number of subjects of both sexes, age 7 to 80 . This was done in order to determine whether there were differences in the number of people classified as having a higher than normal lifelong relative risk of having a fracture, in order to build a normal mestizo reference database that allowed us to define the age at which PBM is established at different skeletal sites. To the best of our knowledge, our study is the largest reported in Latin America, with a data set comprised of healthy, urban mestizos from early childhood to old age, which may represent more than $70 \%$ of the Mexican population. PBM and T-scores derived for this population differ significantly from values derived from commercial manufacturer's US Hispanic database, with our data reflecting significantly higher values and a curve indicating that age-dependent BMD loss is also very different. The T-score derived from our PBM values had only an $86 \%$ concordance with the manufacturers' Hispanic values that classify the normal population using PBM reference values, and up to $89 \%$ for T-score values for the population aged 20 to 40 . Using our reference values, the number of people in the range of osteopenia and osteoporosis increased by 31 and 32 percent, respectively. As expected, this lack of concordance between the Mexican database and the manufacturers' Hispanic database increases as age increases. Revising estimates of fracture risk based on our numbers will have a major clinical impact since fracture prevention interventions will increase. ${ }^{29}$

Since our Mexican database of reference values are not currently included in the software of commercial densitometers used in Mexico, interested users can classify their specific BMD values reported by these instruments according to the reference values presented in the BMD tables of this report (tables III.1-III.2, IV1IV.18). Having normative BMD reference values for the urban Mexican population will be very relevant for our densitometry community. This more accurate normative reference data may give us the opportunity to improve the decision making process used in the prevention and care of bone density-related public health problems, though we must ensure that it does not simply amount to an increased burden on an already strained healthcare system. These considerations should be taken into account by policy makers in order to improve the Mexican healthcare infrastructure as well as to seek new ways to address the growing populations' healthcare needs. 
Table III.I

Bone Mineral Density T score for male population

\begin{tabular}{|c|c|c|c|c|c|c|c|c|c|}
\hline \multirow{2}{*}{$\begin{array}{c}T \\
\text { SCORE }\end{array}$} & \multicolumn{9}{|c|}{ BONE MINERAL DENSITY $\left(\mathrm{g} / \mathrm{cm}^{2}\right)$} \\
\hline & Subtotal body & Lumbar $t=$ region & Hip & Head & Arm & Proximal femur & Trunk & Ribs & Pelvis \\
\hline-5.0 & 0.762 & 0.490 & 0.480 & 0.913 & 0.402 & 0.785 & 0.567 & 0.424 & 0.601 \\
\hline-4.5 & 0.811 & 0.559 & 0.551 & 1.042 & 0.461 & 0.845 & 0.614 & 0.461 & 0.668 \\
\hline-4.0 & 0.861 & 0.629 & 0.621 & 1.171 & 0.520 & 0.906 & 0.662 & 0.498 & 0.735 \\
\hline-3.5 & 0.910 & 0.699 & 0.692 & 1.299 & 0.579 & 0.967 & 0.709 & 0.535 & 0.802 \\
\hline-3.0 & 0.960 & 0.768 & 0.762 & 1.428 & 0.638 & 1.028 & 0.757 & 0.573 & 0.870 \\
\hline-2.5 & 1.009 & 0.838 & 0.832 & 1.557 & 0.697 & 1.088 & 0.804 & 0.610 & 0.937 \\
\hline-2.0 & 1.059 & 0.907 & 0.903 & 1.686 & 0.756 & 1.149 & 0.852 & 0.647 & 1.004 \\
\hline-1.5 & 1.108 & 0.977 & 0.973 & 1.815 & 0.815 & 1.210 & 0.899 & 0.684 & 1.071 \\
\hline-1.0 & 1.158 & 1.047 & 1.044 & 1.944 & 0.874 & 1.270 & 0.946 & 0.722 & 1.138 \\
\hline-0.5 & 1.208 & 1.116 & I.II4 & 2.073 & 0.934 & I.331 & 0.994 & 0.759 & 1.206 \\
\hline 0.0 & 1.257 & 1.186 & 1.185 & 2.202 & 0.993 & 1.392 & 1.041 & 0.796 & 1.273 \\
\hline 0.5 & 1.307 & 1.255 & 1.255 & 2.331 & 1.052 & 1.452 & 1.089 & 0.833 & 1.340 \\
\hline 1.0 & 1.356 & 1.325 & 1.326 & 2.460 & I.III & 1.513 & 1.136 & $0.87 \mid$ & I.407 \\
\hline 1.5 & 1.406 & 1.395 & 1.396 & 2.589 & 1.170 & 1.574 & 1.184 & 0.908 & 1.475 \\
\hline 2.0 & 1.455 & 1.464 & 1.466 & 2.718 & 1.229 & 1.634 & $\mid .231$ & 0.945 & 1.542 \\
\hline 2.5 & 1.505 & 1.534 & 1.537 & 2.847 & 1.288 & 1.695 & 1.279 & 0.982 & 1.609 \\
\hline 3.0 & 1.554 & 1.603 & 1.607 & 2.976 & 1.347 & 1.756 & 1.326 & 1.020 & 1.676 \\
\hline 3.5 & 1.604 & 1.673 & 1.678 & 3.105 & 1.407 & 1.816 & 1.374 & 1.057 & 1.743 \\
\hline 4.0 & 1.653 & 1.743 & 1.748 & 3.234 & 1.466 & I.877 & 1.421 & 1.094 & 1.811 \\
\hline 4.5 & 1.703 & 1.812 & 1.819 & 3.362 & 1.525 & 1.938 & 1.469 & 1.131 & 1.878 \\
\hline 5.0 & 1.752 & 1.882 & 1.889 & 3.491 & 1.584 & 1.999 & 1.516 & 1.168 & 1.945 \\
\hline
\end{tabular}

Table III.2

\section{Bone Mineral Density T score for female population}

\begin{tabular}{|c|c|c|c|c|c|c|c|c|c|}
\hline \multirow{2}{*}{$\begin{array}{c}T \\
\text { SCORE }\end{array}$} & \multicolumn{9}{|c|}{ Bone mineral density $\left(\mathrm{g} / \mathrm{cm}^{2}\right)$} \\
\hline & Subtotal body & Lumbar region & Hip & Head & Arm & Proximal femur & Trunk & Ribs & Pelvis \\
\hline-5.0 & 0.783 & 0.509 & 0.471 & 1.088 & 0.415 & 0.757 & 0.586 & 0.440 & 0.628 \\
\hline-4.5 & 0.823 & 0.576 & 0.531 & 1.225 & 0.460 & 0.800 & 0.625 & 0.470 & 0.681 \\
\hline-4.0 & 0.863 & 0.644 & 0.591 & 1.362 & 0.504 & 0.844 & 0.665 & 0.501 & 0.735 \\
\hline-3.5 & 0.902 & 0.711 & 0.651 & 1.499 & 0.548 & 0.887 & 0.705 & 0.531 & 0.788 \\
\hline-3.0 & 0.942 & 0.778 & 0.711 & 1.636 & 0.592 & 0.931 & 0.745 & 0.562 & $0.84 I$ \\
\hline-2.5 & 0.982 & 0.845 & 0.770 & 1.773 & 0.636 & 0.974 & 0.784 & 0.593 & 0.895 \\
\hline-2.0 & 1.021 & 0.913 & 0.830 & 1.910 & 0.681 & 1.018 & 0.824 & 0.623 & 0.948 \\
\hline-1.5 & 1.061 & 0.980 & 0.890 & 2.047 & 0.725 & 1.061 & 0.864 & 0.654 & 1.002 \\
\hline-1.0 & 1.100 & 1.047 & 0.950 & 2.185 & 0.769 & 1.105 & 0.904 & 0.685 & 1.055 \\
\hline-0.5 & 1.140 & 1.115 & 1.010 & 2.322 & 0.813 & I.I48 & 0.943 & 0.715 & 1.108 \\
\hline 0.0 & 1.180 & 1.182 & 1.070 & 2.459 & 0.857 & 1.192 & 0.983 & 0.746 & 1.162 \\
\hline 0.5 & 1.219 & 1.249 & 1.130 & 2.596 & 0.901 & 1.235 & 1.023 & 0.777 & 1.215 \\
\hline 1.0 & 1.259 & 1.317 & 1.189 & 2.733 & 0.946 & 1.279 & 1.063 & 0.807 & 1.268 \\
\hline 1.5 & 1.299 & 1.384 & 1.249 & 2.870 & 0.990 & 1.323 & 1.102 & 0.838 & 1.322 \\
\hline 2.0 & 1.338 & 1.451 & 1.309 & 3.007 & 1.034 & 1.366 & 1.142 & 0.869 & 1.375 \\
\hline 2.5 & 1.378 & 1.519 & 1.369 & 3.144 & 1.078 & 1.410 & I.182 & 0.899 & 1.429 \\
\hline 3.0 & 1.417 & 1.586 & 1.429 & 3.282 & 1.122 & 1.453 & 1.222 & 0.930 & 1.482 \\
\hline 3.5 & 1.457 & 1.653 & 1.489 & 3.419 & 1.167 & 1.497 & 1.261 & 0.961 & 1.535 \\
\hline 4.0 & 1.497 & 1.720 & 1.548 & 3.556 & 1.211 & 1.540 & 1.301 & 0.991 & 1.589 \\
\hline 4.5 & 1.536 & 1.788 & 1.608 & 3.693 & 1.255 & 1.584 & 1.341 & 1.022 & 1.642 \\
\hline 5.0 & 1.576 & 1.855 & 1.668 & 3.830 & 1.299 & 1.627 & 1.380 & 1.052 & 1.695 \\
\hline
\end{tabular}


Table IV.I

Bone Mineral Density Z score values of subtotal body for male population

\begin{tabular}{|c|c|c|c|c|c|c|c|c|c|c|c|}
\hline \multirow[b]{2}{*}{ Age } & \multicolumn{5}{|c|}{ Z SCORE } & \multirow[b]{2}{*}{ Age } & \multicolumn{5}{|c|}{ Z SCORE } \\
\hline & -2.5 & -1.0 & 0.0 & 1.0 & 2.5 & & -2.5 & -1.0 & 0.0 & 1.0 & 2.5 \\
\hline 7 & 0.524 & 0.673 & 0.772 & 0.871 & 1.019 & 45 & 0.979 & 1.127 & 1.227 & 1.326 & 1.474 \\
\hline 8 & 0.581 & 0.729 & 0.828 & 0.927 & 1.076 & 46 & 0.977 & 1.126 & 1.225 & 1.324 & 1.473 \\
\hline 9 & 0.633 & 0.782 & 0.881 & 0.980 & 1.129 & 47 & 0.976 & 1.125 & 1.224 & 1.323 & $|.47|$ \\
\hline 10 & 0.683 & 0.831 & 0.930 & 1.030 & 1.178 & 48 & 0.975 & 1.123 & 1.222 & 1.321 & 1.470 \\
\hline II & 0.729 & 0.877 & 0.976 & 1.075 & 1.224 & 49 & 0.973 & 1.122 & 1.221 & 1.320 & 1.469 \\
\hline 12 & 0.771 & 0.919 & 1.019 & 1.118 & 1.266 & 50 & 0.972 & 1.121 & 1.220 & 1.319 & I. 467 \\
\hline 13 & 0.810 & 0.958 & 1.057 & 1.156 & 1.305 & 51 & $0.97 \mid$ & 1.119 & 1.218 & 1.317 & 1.466 \\
\hline 14 & 0.845 & 0.993 & 1.092 & 1.191 & 1.340 & 52 & 0.969 & 1.118 & 1.217 & 1.316 & 1.465 \\
\hline 15 & 0.876 & 1.025 & 1.124 & 1.223 & 1.372 & 53 & 0.968 & 1.116 & 1.215 & 1.315 & 1.463 \\
\hline 16 & 0.904 & 1.053 & 1.152 & 1.251 & 1.400 & 54 & 0.966 & 1.115 & 1.214 & 1.313 & 1.462 \\
\hline 17 & 0.929 & 1.077 & 1.176 & 1.275 & I. 424 & 55 & 0.965 & 1.114 & 1.213 & 1.312 & 1.460 \\
\hline 18 & 0.950 & 1.098 & 1.197 & 1.296 & 1.445 & 56 & 0.964 & 1.112 & 1.211 & 1.310 & 1.459 \\
\hline 19 & 0.967 & 1.116 & 1.215 & 1.314 & 1.462 & 57 & 0.962 & 1.111 & 1.210 & 1.309 & 1.458 \\
\hline 20 & 0.981 & 1.129 & 1.228 & 1.328 & 1.476 & 58 & 0.961 & 1.109 & 1.208 & 1.308 & 1.456 \\
\hline 21 & 0.991 & 1.140 & 1.239 & 1.338 & 1.487 & 59 & 0.959 & 1.108 & 1.207 & 1.306 & 1.455 \\
\hline 22 & 0.999 & I.I 48 & 1.247 & 1.346 & 1.495 & 60 & 0.958 & I.107 & 1.206 & 1.305 & 1.453 \\
\hline 23 & 1.005 & 1.153 & 1.252 & 1.351 & 1.500 & 61 & 0.956 & 1.105 & 1.204 & 1.303 & 1.452 \\
\hline 24 & 1.008 & 1.156 & 1.255 & 1.355 & 1.503 & 62 & 0.955 & 1.104 & 1.203 & 1.302 & 1.450 \\
\hline 25 & 1.009 & 1.158 & 1.257 & 1.356 & 1.505 & 63 & 0.953 & 1.102 & 1.201 & 1.300 & 1.449 \\
\hline 26 & 1.009 & 1.158 & 1.257 & 1.356 & 1.505 & 64 & 0.952 & 1.100 & 1.199 & 1.299 & I.447 \\
\hline 27 & 1.008 & I.157 & 1.256 & 1.355 & 1.504 & 65 & 0.950 & 1.099 & 1.198 & 1.297 & 1.445 \\
\hline 28 & 1.007 & I.155 & 1.254 & 1.354 & 1.502 & 66 & 0.948 & I.097 & 1.196 & 1.295 & I.444 \\
\hline 29 & 1.005 & 1.153 & 1.253 & 1.352 & 1.500 & 67 & 0.946 & 1.095 & 1.194 & 1.293 & 1.442 \\
\hline 30 & 1.003 & 1.152 & 1.251 & 1.350 & 1.498 & 68 & 0.944 & 1.093 & 1.192 & 1.291 & 1.439 \\
\hline 31 & 1.001 & 1.150 & 1.249 & 1.348 & 1.496 & 69 & $0.94 I$ & 1.090 & 1.189 & 1.288 & I.437 \\
\hline 32 & 0.999 & I.I 48 & 1.247 & 1.346 & 1.495 & 70 & 0.938 & I.087 & 1.186 & 1.285 & 1.433 \\
\hline 33 & 0.997 & I.I46 & 1.245 & 1.344 & 1.493 & 71 & 0.934 & 1.083 & 1.182 & 1.281 & 1.430 \\
\hline 34 & 0.996 & I.144 & 1.243 & 1.342 & I.49| & 72 & 0.930 & 1.078 & 1.177 & 1.276 & 1.425 \\
\hline 35 & 0.994 & 1.143 & 1.242 & 1.341 & 1.489 & 73 & 0.924 & 1.073 & 1.172 & I.27I & 1.419 \\
\hline 36 & 0.992 & 1.141 & 1.240 & 1.339 & 1.488 & 74 & 0.918 & 1.066 & 1.165 & 1.264 & 1.413 \\
\hline 37 & 0.991 & 1.139 & 1.238 & 1.338 & 1.486 & 75 & 0.910 & 1.059 & 1.158 & 1.257 & 1.406 \\
\hline 38 & 0.989 & 1.138 & 1.237 & 1.336 & 1.485 & 76 & 0.902 & 1.050 & 1.149 & 1.249 & 1.397 \\
\hline 39 & 0.988 & 1.136 & 1.235 & 1.334 & 1.483 & 77 & 0.892 & $|.04|$ & 1.140 & 1.239 & 1.387 \\
\hline 40 & 0.986 & 1.135 & 1.234 & 1.333 & 1.481 & 78 & $0.88 I$ & 1.030 & 1.129 & 1.228 & 1.376 \\
\hline 41 & 0.985 & 1.133 & 1.232 & 1.331 & 1.480 & 79 & 0.869 & 1.017 & 1.116 & 1.215 & 1.364 \\
\hline 42 & 0.983 & 1.132 & 1.231 & 1.330 & 1.478 & 80 & 0.855 & 1.003 & 1.102 & 1.201 & 1.350 \\
\hline 43 & 0.982 & 1.130 & 1.229 & 1.328 & I.477 & & & & & & \\
\hline 44 & 0.980 & 1.129 & 1.228 & 1.327 & 1.476 & & & & & & \\
\hline
\end{tabular}


Table IV.2

\section{Bone Mineral Density Z score values of lumbar region for male population}

\begin{tabular}{|c|c|c|c|c|c|}
\hline \multirow{2}{*}{ Age } & \multicolumn{5}{|c|}{ Z SCORE } \\
\hline & -2.5 & -1.0 & 0.0 & 1.0 & 2.5 \\
\hline 7 & 0.199 & 0.421 & 0.561 & 0.700 & 0.909 \\
\hline 8 & 0.267 & 0.489 & 0.629 & 0.768 & 0.977 \\
\hline 9 & 0.331 & 0.553 & 0.693 & 0.832 & 1.041 \\
\hline 10 & 0.391 & 0.613 & 0.753 & 0.892 & 1.101 \\
\hline II & 0.447 & 0.669 & 0.808 & 0.948 & 1.156 \\
\hline 12 & 0.498 & 0.721 & 0.860 & 0.999 & 1.208 \\
\hline 13 & 0.546 & 0.769 & 0.908 & 1.047 & 1.256 \\
\hline 14 & 0.590 & 0.813 & 0.952 & 1.091 & 1.300 \\
\hline 15 & 0.630 & 0.852 & 0.991 & 1.131 & 1.339 \\
\hline 16 & 0.665 & 0.888 & 1.027 & 1.166 & 1.375 \\
\hline 17 & 0.697 & 0.919 & 1.059 & 1.198 & 1.407 \\
\hline 18 & 0.724 & 0.947 & 1.086 & 1.225 & 1.434 \\
\hline 19 & 0.748 & 0.970 & 1.110 & 1.249 & 1.458 \\
\hline 20 & 0.767 & 0.990 & 1.129 & 1.268 & 1.477 \\
\hline 21 & 0.783 & 1.006 & 1.145 & 1.284 & 1.493 \\
\hline 22 & 0.796 & 1.018 & 1.158 & 1.297 & 1.506 \\
\hline 23 & 0.805 & 1.028 & 1.167 & 1.306 & 1.515 \\
\hline 24 & 0.812 & 1.035 & 1.174 & 1.314 & 1.522 \\
\hline 25 & 0.817 & 1.040 & 1.179 & 1.319 & 1.527 \\
\hline 26 & 0.821 & 1.043 & 1.183 & 1.322 & 1.531 \\
\hline 27 & 0.823 & 1.045 & I.185 & 1.324 & 1.533 \\
\hline 28 & 0.824 & 1.046 & 1.185 & 1.325 & 1.533 \\
\hline 29 & 0.824 & 1.047 & 1.186 & 1.325 & 1.534 \\
\hline 30 & 0.824 & 1.047 & 1.186 & 1.325 & 1.534 \\
\hline 31 & 0.824 & 1.046 & 1.186 & 1.325 & 1.534 \\
\hline 32 & 0.823 & 1.046 & 1.185 & 1.325 & 1.533 \\
\hline 33 & 0.823 & 1.046 & 1.185 & 1.324 & 1.533 \\
\hline 34 & 0.822 & 1.045 & 1.184 & 1.323 & 1.532 \\
\hline 35 & 0.821 & 1.044 & 1.183 & 1.323 & $|.53|$ \\
\hline 36 & 0.821 & 1.043 & 1.182 & 1.322 & 1.530 \\
\hline 37 & 0.819 & 1.042 & 1.181 & 1.321 & 1.529 \\
\hline 38 & 0.818 & 1.041 & 1.180 & 1.319 & 1.528 \\
\hline 39 & 0.817 & 1.040 & 1.179 & 1.318 & 1.527 \\
\hline 40 & 0.816 & 1.038 & 1.178 & 1.317 & 1.526 \\
\hline 41 & 0.814 & 1.037 & 1.176 & 1.315 & 1.524 \\
\hline 42 & 0.813 & 1.036 & 1.175 & 1.314 & 1.523 \\
\hline 43 & 0.811 & 1.034 & 1.173 & 1.312 & 1.521 \\
\hline 44 & 0.810 & 1.032 & 1.172 & 1.311 & 1.520 \\
\hline
\end{tabular}

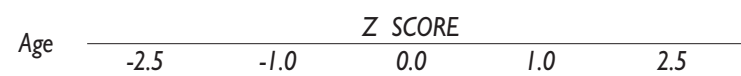

\begin{tabular}{|c|c|c|c|c|c|}
\hline 45 & 0.808 & 1.031 & 1.170 & 1.309 & 1.518 \\
\hline 46 & 0.807 & 1.029 & 1.169 & 1.308 & 1.517 \\
\hline 47 & 0.805 & 1.028 & 1.167 & 1.306 & 1.515 \\
\hline 48 & 0.803 & 1.026 & 1.165 & 1.305 & 1.513 \\
\hline 49 & 0.802 & 1.025 & 1.164 & 1.303 & 1.512 \\
\hline 50 & 0.800 & 1.023 & 1.162 & 1.302 & 1.510 \\
\hline 51 & 0.799 & 1.022 & 1.161 & 1.300 & 1.509 \\
\hline 52 & 0.798 & 1.020 & 1.160 & 1.299 & 1.508 \\
\hline 53 & 0.796 & 1.019 & 1.158 & 1.298 & 1.506 \\
\hline 54 & 0.795 & 1.018 & I.157 & 1.296 & 1.505 \\
\hline 55 & 0.794 & 1.017 & 1.156 & 1.295 & 1.504 \\
\hline 56 & 0.793 & 1.016 & 1.155 & 1.294 & 1.503 \\
\hline 57 & 0.792 & 1.015 & 1.154 & 1.294 & 1.502 \\
\hline 58 & 0.792 & 1.015 & 1.154 & 1.293 & 1.502 \\
\hline 59 & 0.791 & 1.014 & 1.153 & 1.292 & 1.501 \\
\hline 60 & 0.791 & 1.014 & 1.153 & 1.292 & 1.501 \\
\hline 61 & 0.791 & 1.014 & 1.153 & 1.292 & 1.501 \\
\hline 62 & 0.791 & 1.014 & 1.153 & 1.292 & 1.501 \\
\hline 63 & 0.791 & 1.014 & 1.153 & 1.292 & I.50I \\
\hline 64 & 0.792 & 1.014 & 1.154 & 1.293 & 1.502 \\
\hline 65 & 0.792 & 1.015 & 1.154 & 1.293 & 1.502 \\
\hline 66 & 0.793 & 1.016 & 1.155 & 1.294 & 1.503 \\
\hline 67 & 0.793 & 1.016 & 1.155 & 1.294 & 1.503 \\
\hline 68 & 0.793 & 1.016 & 1.155 & 1.294 & 1.503 \\
\hline 69 & 0.792 & 1.015 & 1.154 & 1.294 & 1.502 \\
\hline 70 & 0.790 & 1.013 & 1.152 & 1.292 & 1.500 \\
\hline 71 & 0.787 & 1.010 & 1.149 & 1.288 & I.497 \\
\hline 72 & 0.783 & 1.005 & 1.145 & 1.284 & 1.493 \\
\hline 73 & 0.776 & 0.999 & 1.138 & 1.277 & 1.486 \\
\hline 74 & 0.768 & 0.991 & 1.130 & 1.269 & 1.478 \\
\hline 75 & 0.757 & 0.980 & 1.119 & 1.258 & I.467 \\
\hline 76 & 0.744 & 0.967 & 1.106 & 1.245 & 1.454 \\
\hline 77 & 0.728 & 0.951 & 1.090 & 1.229 & 1.438 \\
\hline 78 & 0.709 & 0.932 & 1.071 & 1.210 & 1.419 \\
\hline 79 & 0.687 & 0.909 & 1.049 & 1.188 & 1.397 \\
\hline 80 & 0.661 & 0.884 & 1.023 & 1.162 & 1.371 \\
\hline
\end{tabular}


Table IV.3

Bone Mineral Density Z score values of hip for male population

\begin{tabular}{|c|c|c|c|c|c|}
\hline \multirow{2}{*}{ Age } & \multicolumn{5}{|c|}{ Z SCORE } \\
\hline & -2.5 & -1.0 & 0.0 & 1.0 & 2.5 \\
\hline 7 & 0.374 & 0.585 & 0.726 & 0.867 & 1.078 \\
\hline 8 & 0.434 & 0.645 & 0.786 & 0.927 & 1.138 \\
\hline 9 & 0.490 & 0.701 & 0.842 & 0.983 & 1.195 \\
\hline 10 & 0.542 & 0.753 & 0.894 & 1.035 & 1.246 \\
\hline II & 0.589 & 0.801 & 0.942 & 1.083 & 1.294 \\
\hline 12 & 0.633 & 0.844 & 0.985 & 1.126 & 1.337 \\
\hline 13 & 0.672 & 0.883 & 1.024 & 1.165 & 1.376 \\
\hline 14 & 0.706 & 0.918 & 1.059 & 1.199 & 1.411 \\
\hline 15 & 0.737 & 0.948 & 1.089 & 1.230 & |.44I \\
\hline 16 & 0.763 & 0.974 & 1.115 & 1.256 & 1.467 \\
\hline 17 & 0.785 & 0.996 & 1.137 & 1.278 & 1.489 \\
\hline 18 & 0.802 & 1.014 & 1.155 & 1.296 & 1.507 \\
\hline 19 & 0.816 & 1.027 & 1.168 & 1.309 & 1.520 \\
\hline 20 & 0.825 & 1.036 & 1.177 & 1.318 & 1.529 \\
\hline 21 & 0.830 & 1.042 & 1.183 & 1.323 & 1.535 \\
\hline 22 & 0.832 & 1.044 & 1.185 & 1.326 & 1.537 \\
\hline 23 & 0.832 & 1.043 & 1.184 & 1.325 & 1.536 \\
\hline 24 & 0.829 & 1.040 & 1.181 & 1.322 & 1.533 \\
\hline 25 & 0.824 & 1.035 & 1.176 & 1.317 & 1.528 \\
\hline 26 & 0.817 & 1.029 & 1.170 & 1.310 & 1.522 \\
\hline 27 & 0.810 & 1.021 & 1.162 & 1.303 & 1.515 \\
\hline 28 & 0.803 & 1.014 & 1.155 & 1.296 & 1.507 \\
\hline 29 & 0.795 & 1.006 & I.I47 & 1.288 & 1.499 \\
\hline 30 & 0.788 & 0.999 & 1.140 & 1.281 & 1.492 \\
\hline 31 & $0.78 I$ & 0.993 & 1.134 & 1.275 & 1.486 \\
\hline 32 & 0.776 & 0.987 & 1.128 & 1.269 & 1.480 \\
\hline 33 & 0.770 & 0.981 & 1.122 & 1.263 & 1.475 \\
\hline 34 & 0.765 & 0.976 & 1.117 & 1.258 & 1.470 \\
\hline 35 & 0.760 & 0.972 & 1.113 & 1.254 & 1.465 \\
\hline 36 & 0.756 & 0.968 & 1.109 & 1.249 & $|.46|$ \\
\hline 37 & 0.753 & 0.964 & 1.105 & 1.246 & 1.457 \\
\hline 38 & 0.749 & 0.960 & 1.101 & 1.242 & 1.454 \\
\hline 39 & 0.746 & 0.957 & 1.098 & 1.239 & 1.451 \\
\hline 40 & 0.743 & 0.955 & 1.095 & 1.236 & 1.448 \\
\hline 41 & $0.74 I$ & 0.952 & 1.093 & 1.234 & 1.445 \\
\hline 42 & 0.738 & 0.950 & 1.091 & 1.232 & 1.443 \\
\hline 43 & 0.736 & 0.948 & 1.089 & 1.230 & $|.44|$ \\
\hline 44 & 0.735 & 0.946 & 1.087 & 1.228 & 1.439 \\
\hline
\end{tabular}

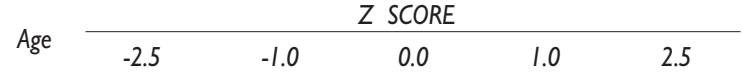

\begin{tabular}{llllll}
45 & 0.733 & 0.944 & 1.085 & 1.226 & 1.437 \\
\hline 46 & 0.731 & 0.943 & 1.084 & 1.225 & 1.436 \\
\hline 47 & 0.730 & 0.941 & 1.082 & 1.223 & 1.435 \\
\hline 48 & 0.729 & 0.940 & 1.081 & 1.222 & 1.433 \\
\hline 49 & 0.728 & 0.939 & 1.080 & 1.221 & 1.432 \\
\hline
\end{tabular}

\begin{tabular}{llllll}
\hline 50 & 0.726 & 0.938 & 1.079 & 1.219 & 1.431 \\
\hline 51 & 0.725 & 0.937 & 1.077 & 1.218 & 1.430 \\
\hline 52 & 0.724 & 0.935 & 1.076 & 1.217 & 1.428
\end{tabular}

\begin{tabular}{llllll}
\hline 53 & 0.723 & 0.934 & 1.075 & 1.216 & 1.427 \\
\hline 54 & 0.721 & 0.933 & 1.074 & 1.215 & 1.426 \\
\hline 55 & 0.720 & 0.931 & 1.072 & 1.213 & 1.425 \\
\hline 56 & 0.719 & 0.930 & 1.071 & 1.212 & 1.423
\end{tabular}

\begin{tabular}{llllll}
\hline 57 & 0.717 & 0.928 & 1.069 & 1.210 & 1.421 \\
\hline 58 & 0.715 & 0.927 & 1.067 & 1.208 & 1.420 \\
\hline 59 & 0.713 & 0.925 & 1.065 & 1.206 & 1.418 \\
\hline 60 & 0.711 & 0.922 & 1.063 & 1.204 & 1.415
\end{tabular}

\begin{tabular}{llllll}
\hline 60 & 0.711 & 0.922 & 1.063 & 1.204 & 1.415 \\
\hline 61 & 0.708 & 0.920 & 1.061 & 1.202 & 1.413
\end{tabular}

\begin{tabular}{llllll}
\hline 62 & 0.706 & 0.917 & 1.058 & 1.199 & 1.410 \\
\hline
\end{tabular}

\begin{tabular}{llllll}
\hline 63 & 0.703 & 0.914 & 1.055 & 1.196 & 1.407 \\
\hline 64 & 0.699 & 0.911 & 1.051 & 1.192 & 1.404
\end{tabular}

\begin{tabular}{llllll}
\hline 65 & 0.695 & 0.907 & 1.048 & 1.189 & 1.400 \\
\hline 66 & 0.691 & 0.903 & 1.044 & 1.184 & 1.396
\end{tabular}

\begin{tabular}{llllll}
66 & 0.691 & 0.903 & 1.044 & 1.184 & 1.396 \\
\hline 67 & 0.687 & 0.898 & 1.039 & 1.180 & 1.391 \\
\hline 68 & 0.681 & 0.893 & 1.034 & 1.175 & 1.386 \\
\hline 69 & 0.676 & 0.887 & 1.028 & 1.169 & 1.380
\end{tabular}

\begin{tabular}{llllll}
69 & 0.676 & 0.887 & 1.028 & 1.169 & 1.380 \\
\hline 70 & 0.669 & 0.880 & 1.021 & 1.162 & 1.374 \\
\hline 71 & 0.662 & 0.873 & 1.014 & 1.155 & 1.366 \\
\hline
\end{tabular}

\begin{tabular}{llllll}
\hline 72 & 0.654 & 0.865 & 1.006 & 1.147 & 1.358 \\
\hline
\end{tabular}

\begin{tabular}{llllll}
\hline 73 & 0.645 & 0.856 & 0.997 & 1.138 & 1.349 \\
\hline 74 & 0.635 & 0.846 & 0.987 & 1.128 & 1.339 \\
\hline 75 & 0.624 & 0.835 & 0.976 & 1.117 & 1.328 \\
\hline 76 & 0.612 & 0.823 & 0.964 & 1.105 & 1.316 \\
\hline 77 & 0.599 & 0.810 & 0.951 & 1.092 & 1.303 \\
\hline 78 & 0.584 & 0.796 & 0.936 & 1.077 & 1.289 \\
\hline 79 & 0.568 & 0.780 & 0.921 & 1.062 & 1.273 \\
\hline 80 & 0.551 & 0.763 & 0.904 & 1.045 & 1.256
\end{tabular}


Table IV. 4

Bone Mineral Density Z score values of head for male population

\begin{tabular}{|c|c|c|c|c|c|}
\hline \multirow{2}{*}{ Age } & \multicolumn{5}{|c|}{ Z SCORE } \\
\hline & -2.5 & -1.0 & 0.0 & 1.0 & 2.5 \\
\hline 7 & 0.696 & 1.083 & $1.34 \mid$ & 1.599 & 1.986 \\
\hline 8 & 0.780 & 1.167 & 1.425 & 1.683 & 2.070 \\
\hline 9 & 0.860 & 1.246 & 1.504 & 1.762 & 2.149 \\
\hline 10 & 0.934 & 1.321 & 1.579 & 1.837 & 2.224 \\
\hline II & 1.005 & 1.392 & 1.649 & 1.907 & 2.294 \\
\hline 12 & 1.070 & 1.457 & 1.715 & 1.973 & 2.360 \\
\hline 13 & 1.131 & 1.518 & 1.776 & 2.034 & 2.421 \\
\hline 14 & 1.188 & 1.575 & 1.833 & 2.091 & 2.477 \\
\hline 15 & 1.240 & 1.627 & 1.885 & 2.143 & 2.529 \\
\hline 16 & 1.287 & 1.674 & 1.932 & 2.190 & 2.577 \\
\hline 17 & 1.330 & 1.717 & 1.975 & 2.233 & 2.619 \\
\hline 18 & 1.368 & 1.755 & 2.013 & 2.271 & 2.658 \\
\hline 19 & 1.402 & 1.789 & 2.047 & 2.305 & 2.691 \\
\hline 20 & 1.431 & 1.818 & 2.076 & 2.334 & 2.721 \\
\hline 21 & 1.456 & 1.843 & 2.101 & 2.359 & 2.745 \\
\hline 22 & 1.477 & 1.864 & 2.122 & 2.380 & 2.767 \\
\hline 23 & 1.495 & 1.882 & 2.139 & 2.397 & 2.784 \\
\hline 24 & 1.509 & 1.896 & 2.154 & 2.412 & 2.799 \\
\hline 25 & 1.521 & 1.908 & 2.166 & 2.424 & 2.810 \\
\hline 26 & 1.530 & 1.917 & 2.175 & 2.433 & 2.820 \\
\hline 27 & 1.538 & 1.925 & 2.182 & 2.440 & 2.827 \\
\hline 28 & 1.544 & 1.930 & 2.188 & 2.446 & 2.833 \\
\hline 29 & 1.548 & 1.935 & 2.193 & 2.451 & 2.837 \\
\hline 30 & 1.551 & 1.938 & 2.196 & 2.454 & $2.84 I$ \\
\hline 31 & 1.554 & 1.941 & 2.199 & 2.457 & 2.843 \\
\hline 32 & 1.556 & 1.943 & 2.201 & 2.458 & 2.845 \\
\hline 33 & 1.557 & 1.944 & 2.202 & 2.459 & 2.846 \\
\hline 34 & 1.557 & 1.944 & 2.202 & 2.460 & 2.847 \\
\hline 35 & 1.557 & 1.944 & 2.202 & 2.460 & 2.846 \\
\hline 36 & 1.556 & 1.943 & 2.201 & 2.459 & 2.846 \\
\hline 37 & 1.555 & 1.942 & 2.200 & 2.458 & 2.844 \\
\hline 38 & 1.553 & 1.940 & 2.198 & 2.456 & 2.843 \\
\hline 39 & $|.55|$ & 1.938 & 2.196 & 2.454 & $2.84 I$ \\
\hline 40 & 1.549 & 1.936 & 2.194 & $2.45 I$ & 2.838 \\
\hline 41 & 1.546 & 1.933 & 2.191 & 2.449 & 2.836 \\
\hline 42 & 1.543 & 1.930 & 2.188 & 2.446 & 2.833 \\
\hline 43 & 1.540 & 1.927 & 2.185 & 2.443 & 2.830 \\
\hline 44 & 1.537 & 1.924 & 2.182 & 2.440 & 2.827 \\
\hline
\end{tabular}

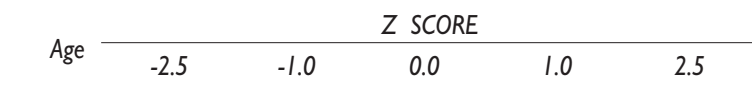

\begin{tabular}{llllll}
45 & 1.534 & 1.921 & 2.179 & 2.437 & 2.824 \\
\hline 46 & 1.531 & 1.918 & 2.176 & 2.434 & 2.820 \\
\hline 47 & 1.528 & 1.915 & 2.173 & 2.431 & 2.818 \\
\hline
\end{tabular}

\begin{tabular}{llllll}
\hline 48 & 1.525 & 1.912 & 2.170 & 2.428 & 2.815 \\
\hline 49 & 1.523 & 1.910 & 2.167 & 2.425 & 2.812 \\
\hline 50 & 1.520 & 1.907 & 2.165 & 2.423 & 2.810
\end{tabular}

\begin{tabular}{llllll}
\hline 50 & 1.520 & 1.907 & 2.165 & 2.423 & 2.810 \\
\hline 51 & 1.518 & 1.905 & 2.163 & 2.421 & 2.808 \\
\hline 52 & 1.517 & 1.904 & 2.162 & 2.419 & 2.806 \\
\hline
\end{tabular}

\begin{tabular}{rrrrrr}
52 & 1.517 & 1.904 & 2.162 & 2.419 & 2.806 \\
\hline 53 & 1.516 & 1.903 & 2.160 & 2.418 & 2.805 \\
\hline 54 & 1.515 & 1.902 & 2.160 & 2.418 & 2.804 \\
\hline 55 & 1.515 & 1.902 & 2.160 & 2.418 & 2.804
\end{tabular}

\begin{tabular}{llllll}
55 & 1.515 & 1.902 & 2.160 & 2.418 & 2.804 \\
\hline 56 & 1.516 & 1.902 & 2.160 & 2.418 & 2.805 \\
\hline 57 & 1.517 & 1.904 & 2.162 & 2.419 & 2.806 \\
\hline 58 & 1.519 & 1.906 & 2.164 & 2.421 & 2.808 \\
\hline 59 & 1.522 & 1.909 & 2.166 & 2.424 & 2.811 \\
\hline
\end{tabular}

\begin{tabular}{llllll}
\hline 59 & 1.522 & 1.909 & 2.166 & 2.424 & 2.811 \\
\hline 60 & 1.525 & 1.912 & 2.170 & 2.428 & 2.815 \\
\hline 61 & 1.530 & 1.917 & 2.175 & 2.433 & 2.819 \\
\hline 62 & 1.536 & 1.923 & 2.180 & 2.438 & 2.825 \\
\hline 63 & 1.542 & 1.929 & 2.187 & 2.445 & 2.832
\end{tabular}

\begin{tabular}{llllll}
\hline 63 & 1.542 & 1.929 & 2.187 & 2.445 & 2.832 \\
\hline 64 & 1.550 & 1.937 & 2.195 & 2.453 & 2.840 \\
\hline 65 & 1.559 & 1.946 & 2.204 & 2.462 & 2.848 \\
\hline 66 & 1.568 & 1.955 & 2.213 & 2.471 & 2.858 \\
\hline 67 & 1.577 & 1.964 & 2.222 & 2.480 & 2.867 \\
\hline 68 & 1.585 & 1.972 & 2.230 & 2.488 & 2.875 \\
\hline 69 & 1.591 & 1.978 & 2.236 & 2.494 & 2.881 \\
\hline 70 & 1.595 & 1.982 & 2.240 & 2.498 & 2.884 \\
\hline 71 & 1.595 & 1.982 & 2.240 & 2.498 & 2.885 \\
\hline 72 & 1.592 & 1.979 & 2.236 & 2.494 & 2.881 \\
\hline 73 & 1.583 & 1.970 & 2.228 & 2.486 & 2.873 \\
\hline 74 & 1.570 & 1.957 & 2.214 & 2.472 & 2.859 \\
\hline 75 & 1.550 & 1.937 & 2.194 & 2.452 & 2.839 \\
\hline 76 & 1.523 & 1.910 & 2.168 & 2.425 & 2.812 \\
\hline 77 & 1.488 & 1.875 & 2.133 & 2.391 & 2.778 \\
\hline 78 & 1.445 & 1.832 & 2.090 & 2.348 & 2.735 \\
\hline 79 & 1.393 & 1.780 & 2.038 & 2.296 & 2.683 \\
\hline 80 & 1.331 & 1.718 & 1.976 & 2.234 & 2.621 \\
\hline & & & & & \\
\hline
\end{tabular}


Table IV.5

Bone Mineral Density Z score values of arm for male population

\begin{tabular}{|c|c|c|c|c|c|}
\hline \multirow{2}{*}{ Age } & \multicolumn{5}{|c|}{ Z SCORE } \\
\hline & -2.5 & -1.0 & 0.0 & 1.0 & 2.5 \\
\hline 7 & 0.218 & 0.396 & 0.514 & 0.632 & 0.809 \\
\hline 8 & 0.270 & 0.448 & 0.566 & 0.684 & 0.862 \\
\hline 9 & 0.319 & 0.497 & 0.615 & 0.733 & 0.911 \\
\hline 10 & 0.365 & 0.543 & 0.661 & 0.779 & 0.957 \\
\hline II & 0.408 & 0.586 & 0.704 & 0.822 & 0.999 \\
\hline 12 & 0.448 & 0.625 & 0.744 & 0.862 & 1.039 \\
\hline 13 & 0.485 & 0.662 & 0.780 & 0.898 & 1.076 \\
\hline 14 & 0.518 & 0.695 & 0.814 & 0.932 & 1.109 \\
\hline 15 & 0.548 & 0.726 & 0.844 & 0.962 & 1.140 \\
\hline 16 & 0.576 & 0.753 & $0.87 \mid$ & 0.990 & 1.167 \\
\hline 17 & 0.600 & 0.777 & 0.895 & 1.014 & 1.191 \\
\hline 18 & 0.621 & 0.798 & 0.916 & 1.035 & 1.212 \\
\hline 19 & 0.639 & 0.816 & 0.934 & 1.053 & 1.230 \\
\hline 20 & 0.654 & 0.831 & 0.949 & 1.067 & 1.245 \\
\hline 21 & 0.666 & 0.843 & 0.961 & 1.079 & 1.257 \\
\hline 22 & 0.675 & 0.852 & $0.97 \mid$ & 1.089 & 1.266 \\
\hline 23 & 0.682 & 0.860 & 0.978 & 1.096 & 1.274 \\
\hline 24 & 0.688 & 0.865 & 0.983 & 1.102 & 1.279 \\
\hline 25 & 0.691 & 0.869 & 0.987 & 1.105 & 1.283 \\
\hline 26 & 0.694 & $0.87 \mid$ & 0.990 & 1.108 & 1.285 \\
\hline 27 & 0.695 & 0.873 & 0.991 & 1.109 & 1.287 \\
\hline 28 & 0.696 & 0.874 & 0.992 & 1.110 & 1.287 \\
\hline 29 & 0.697 & 0.874 & 0.992 & 1.110 & 1.288 \\
\hline 30 & 0.697 & 0.874 & 0.992 & I.III & 1.288 \\
\hline 31 & 0.697 & 0.874 & 0.993 & I.III & 1.288 \\
\hline 32 & 0.697 & 0.874 & 0.993 & I.III & 1.288 \\
\hline 33 & 0.697 & 0.874 & 0.993 & I.III & 1.288 \\
\hline 34 & 0.697 & 0.874 & 0.993 & I.III & 1.288 \\
\hline 35 & 0.697 & 0.874 & 0.992 & I.III & 1.288 \\
\hline 36 & 0.696 & 0.874 & 0.992 & 1.110 & 1.288 \\
\hline 37 & 0.696 & 0.873 & 0.992 & 1.110 & 1.287 \\
\hline 38 & 0.695 & 0.873 & 0.991 & 1.109 & 1.287 \\
\hline 39 & 0.695 & 0.872 & 0.990 & 1.109 & 1.286 \\
\hline 40 & 0.694 & $0.87 \mid$ & 0.990 & 1.108 & 1.285 \\
\hline $4 I$ & 0.693 & $0.87 \mid$ & 0.989 & I.107 & 1.284 \\
\hline 42 & 0.692 & 0.870 & 0.988 & 1.106 & 1.283 \\
\hline 43 & 0.691 & 0.868 & 0.987 & 1.105 & 1.282 \\
\hline 44 & 0.690 & 0.867 & 0.985 & 1.104 & 1.281 \\
\hline
\end{tabular}

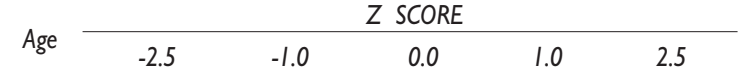

\begin{tabular}{|c|c|c|c|c|c|}
\hline 45 & 0.688 & 0.866 & 0.984 & 1.102 & 1.280 \\
\hline 46 & 0.687 & 0.864 & 0.982 & 1.101 & 1.278 \\
\hline 47 & 0.685 & 0.863 & 0.981 & 1.099 & 1.276 \\
\hline 48 & 0.683 & 0.861 & 0.979 & 1.097 & 1.275 \\
\hline 49 & 0.681 & 0.859 & 0.977 & 1.095 & 1.273 \\
\hline 50 & 0.679 & 0.857 & 0.975 & 1.093 & I.27I \\
\hline 51 & 0.677 & 0.855 & 0.973 & 1.091 & 1.268 \\
\hline 52 & 0.675 & 0.852 & 0.970 & 1.089 & 1.266 \\
\hline 53 & 0.672 & 0.850 & 0.968 & 1.086 & 1.263 \\
\hline 54 & 0.670 & 0.847 & 0.965 & 1.083 & 1.261 \\
\hline 55 & 0.667 & 0.844 & 0.962 & 1.081 & 1.258 \\
\hline 56 & 0.664 & $0.84 I$ & 0.959 & 1.078 & 1.255 \\
\hline 57 & 0.661 & 0.838 & 0.956 & 1.074 & 1.252 \\
\hline 58 & 0.657 & 0.835 & 0.953 & 1.071 & 1.248 \\
\hline 59 & 0.654 & 0.831 & 0.949 & 1.068 & 1.245 \\
\hline 60 & 0.650 & 0.827 & 0.946 & 1.064 & 1.241 \\
\hline 61 & 0.646 & 0.824 & 0.942 & 1.060 & 1.237 \\
\hline 62 & 0.642 & 0.820 & 0.938 & 1.056 & 1.233 \\
\hline 63 & 0.638 & 0.815 & 0.934 & 1.052 & 1.229 \\
\hline 64 & 0.634 & 0.811 & 0.929 & 1.048 & 1.225 \\
\hline 65 & 0.629 & 0.807 & 0.925 & 1.043 & 1.220 \\
\hline 66 & 0.625 & 0.802 & 0.920 & 1.038 & 1.216 \\
\hline 67 & 0.620 & 0.797 & 0.916 & 1.034 & 1.211 \\
\hline 68 & 0.615 & 0.793 & 0.911 & 1.029 & 1.207 \\
\hline 69 & 0.611 & 0.788 & 0.907 & 1.025 & 1.202 \\
\hline 70 & 0.607 & 0.784 & 0.902 & 1.021 & 1.198 \\
\hline 71 & 0.603 & $0.78 I$ & 0.899 & 1.017 & 1.194 \\
\hline 72 & 0.600 & 0.777 & 0.895 & 1.014 & 1.191 \\
\hline 73 & 0.597 & 0.775 & 0.893 & 1.011 & 1.188 \\
\hline 74 & 0.595 & 0.772 & 0.891 & 1.009 & 1.186 \\
\hline 75 & 0.594 & $0.77 I$ & 0.889 & 1.008 & 1.185 \\
\hline 76 & 0.591 & 0.769 & 0.887 & 1.005 & 1.183 \\
\hline 77 & 0.589 & 0.767 & 0.885 & 1.003 & 1.181 \\
\hline 78 & 0.587 & 0.765 & 0.883 & 1.001 & 1.179 \\
\hline 79 & 0.585 & 0.763 & 0.881 & 0.999 & 1.177 \\
\hline 80 & 0.583 & 0.761 & 0.879 & 0.997 & 1.175 \\
\hline
\end{tabular}


Table IV.6

\section{Bone Mineral Density Z score values of proximal femur for male population}

\begin{tabular}{|c|c|c|c|c|c|}
\hline \multirow{2}{*}{ Age } & \multicolumn{5}{|c|}{ Z SCORE } \\
\hline & -2.5 & -1.0 & 0.0 & 1.0 & 2.5 \\
\hline 7 & 0.408 & 0.590 & 0.711 & 0.832 & 1.014 \\
\hline 8 & 0.493 & 0.675 & 0.797 & 0.918 & 1.100 \\
\hline 9 & 0.573 & 0.755 & 0.876 & 0.998 & 1.180 \\
\hline 10 & 0.647 & 0.829 & 0.950 & 1.072 & 1.254 \\
\hline 11 & 0.715 & 0.897 & 1.019 & 1.140 & 1.322 \\
\hline 12 & 0.777 & 0.960 & 1.081 & 1.202 & 1.384 \\
\hline 13 & 0.834 & 1.016 & 1.137 & 1.259 & $1.44 \mid$ \\
\hline 14 & 0.885 & 1.067 & I. 188 & 1.310 & 1.492 \\
\hline 15 & 0.930 & 1.112 & 1.233 & 1.355 & 1.537 \\
\hline 16 & 0.969 & 1.151 & 1.273 & 1.394 & 1.576 \\
\hline 17 & 1.002 & 1.185 & 1.306 & 1.427 & 1.609 \\
\hline 18 & 1.030 & 1.212 & 1.334 & 1.455 & 1.637 \\
\hline 19 & 1.052 & 1.234 & 1.355 & 1.477 & 1.659 \\
\hline 20 & 1.068 & 1.250 & 1.372 & 1.493 & 1.675 \\
\hline 21 & 1.079 & 1.261 & 1.383 & 1.504 & 1.686 \\
\hline 22 & 1.086 & 1.268 & 1.389 & 1.510 & 1.692 \\
\hline 23 & 1.088 & 1.270 & 1.392 & 1.513 & 1.695 \\
\hline 24 & 1.088 & 1.270 & 1.391 & 1.512 & 1.694 \\
\hline 25 & 1.084 & 1.266 & 1.388 & 1.509 & 1.691 \\
\hline 26 & 1.079 & 1.261 & 1.383 & 1.504 & 1.686 \\
\hline 27 & 1.073 & 1.255 & 1.376 & 1.498 & 1.680 \\
\hline 28 & 1.066 & 1.248 & 1.369 & 1.490 & 1.672 \\
\hline 29 & 1.058 & 1.240 & 1.362 & 1.483 & 1.665 \\
\hline 30 & 1.052 & 1.234 & 1.355 & 1.477 & 1.659 \\
\hline 31 & 1.046 & 1.228 & 1.349 & 1.470 & 1.653 \\
\hline 32 & 1.040 & 1.222 & 1.344 & 1.465 & 1.647 \\
\hline 33 & 1.035 & 1.217 & 1.339 & 1.460 & 1.642 \\
\hline 34 & 1.031 & 1.213 & 1.334 & 1.455 & 1.637 \\
\hline 35 & 1.026 & 1.209 & 1.330 & $\mid .451$ & 1.633 \\
\hline 36 & 1.023 & 1.205 & 1.326 & 1.448 & 1.630 \\
\hline 37 & 1.020 & 1.202 & 1.323 & 1.444 & 1.626 \\
\hline 38 & 1.017 & 1.199 & 1.320 & I.44I & 1.624 \\
\hline 39 & 1.014 & 1.196 & 1.318 & 1.439 & 1.621 \\
\hline 40 & 1.012 & 1.194 & 1.315 & 1.437 & 1.619 \\
\hline 41 & 1.010 & 1.192 & 1.313 & 1.435 & 1.617 \\
\hline 42 & 1.008 & 1.190 & 1.312 & 1.433 & 1.615 \\
\hline 43 & 1.007 & 1.189 & 1.310 & 1.432 & 1.614 \\
\hline 44 & 1.006 & 1.188 & 1.309 & 1.430 & 1.613 \\
\hline
\end{tabular}

\begin{tabular}{ccccc} 
Z Z SCORE \\
\cline { 2 - 5 } & -2.5 & -1.0 & 0.0 & 1.0
\end{tabular}

\begin{tabular}{llllll}
45 & 1.005 & 1.187 & 1.308 & 1.429 & 1.611 \\
\hline 46 & 1.004 & 1.186 & 1.307 & 1.429 & 1.611 \\
\hline 47 & 1.003 & 1.185 & 1.306 & 1.428 & 1.610 \\
\hline 48 & 1.002 & 1.184 & 1.306 & 1.427 & 1.609 \\
\hline 49 & 1.001 & 1.184 & 1.305 & 1.426 & 1.608 \\
\hline 50 & 1.001 & 1.183 & 1.304 & 1.426 & 1.608 \\
\hline 51 & 1.000 & 1.182 & 1.304 & 1.425 & 1.607
\end{tabular}

\begin{tabular}{llllll}
\hline 52 & 0.999 & 1.182 & 1.303 & 1.424 & 1.606 \\
\hline 53 & 0.999 & 1.181 & 1.302 & 1.424 & 1.606 \\
\hline 54 & 0.998 & 1.180 & 1.301 & 1.423 & 1.605
\end{tabular}

\begin{tabular}{llllll}
55 & 0.998 & 1.180 & 1.301 & 1.423 & 1.605 \\
\hline 56 & 0.997 & 1.179 & 1.300 & 1.422 & 1.604 \\
\hline 5 & 0.996 & 1.178 & 1.299 & 1.421 & 1.603
\end{tabular}

\begin{tabular}{rlllll}
\hline 56 & 0.996 & 1.178 & 1.299 & 1.421 & 1.603 \\
\hline 57 & 0.994 & 1.177 & 1.298 & 1.419 & 1.601 \\
\hline 58 & 0.993 & 1.175 & 1.296 & 1.418 & 1.600
\end{tabular}

\begin{tabular}{llllll}
\hline 59 & 0.991 & 1.173 & 1.295 & 1.416 & 1.598 \\
\hline 60 & 0.989 & 1.171 & 1.293 & 1.414 & 1.596 \\
\hline 61 & 0.987 & 1.169 & 1.290 & 1.412 & 1.594
\end{tabular}

\begin{tabular}{llllll}
61 & 0.987 & 1.169 & 1.290 & 1.412 & 1.594 \\
\hline 62 & 0.984 & 1.166 & 1.288 & 1.409 & 1.591 \\
\hline 63 & 0.981 & 1.163 & 1.285 & 1.406 & 1.588 \\
\hline 64 & 0.978 & 1.160 & 1.281 & 1.403 & 1.585 \\
\hline
\end{tabular}

\begin{tabular}{llllll}
\hline 65 & 0.974 & 1.156 & 1.278 & 1.399 & 1.581 \\
\hline 66 & 0.970 & 1.152 & 1.273 & 1.395 & 1.577 \\
\hline 67 & 0.965 & 1.148 & 1.269 & 1.390 & 1.572
\end{tabular}

\begin{tabular}{llllll}
\hline 68 & 0.961 & 1.143 & 1.264 & 1.385 & 1.567 \\
\hline 69 & 0.955 & 1.138 & 1.259 & 1.380 & 1.562
\end{tabular}

\begin{tabular}{llllll}
\hline 69 & 0.955 & 1.138 & 1.259 & 1.380 & 1.562 \\
\hline 70 & 0.950 & 1.132 & 1.253 & 1.375 & 1.557 \\
\hline 71 & 0.944 & 1.126 & 1.248 & 1.369 & 1.551 \\
\hline 72 & 0.938 & 1.120 & 1.242 & 1.363 & 1.545 \\
\hline 73 & 0.932 & 1.114 & 1.236 & 1.357 & 1.539 \\
\hline 74 & 0.926 & 1.108 & 1.229 & 1.351 & 1.533 \\
\hline 75 & 0.919 & 1.102 & 1.223 & 1.344 & 1.526 \\
\hline 76 & 0.913 & 1.095 & 1.216 & 1.338 & 1.520 \\
\hline 77 & 0.906 & 1.088 & 1.210 & 1.331 & 1.513 \\
\hline 78 & 0.899 & 1.082 & 1.203 & 1.324 & 1.506 \\
\hline 79 & 0.893 & 1.075 & 1.196 & 1.318 & 1.500 \\
\hline 80 & 0.886 & 1.068 & 1.189 & 1.311 & 1.493
\end{tabular}


Table IV.7

Bone Mineral Density Z score values of trunk for male population

\begin{tabular}{|c|c|c|c|c|c|c|c|c|c|c|c|}
\hline \multirow{2}{*}{ Age } & \multicolumn{5}{|c|}{ Z SCORE } & \multirow{2}{*}{ Age } & \multicolumn{5}{|c|}{ Z SCORE } \\
\hline & -2.5 & -1.0 & 0.0 & 1.0 & 2.5 & & -2.5 & -1.0 & 0.0 & 1.0 & 2.5 \\
\hline 7 & $0.34 I$ & 0.483 & 0.578 & 0.673 & 0.816 & 45 & 0.776 & 0.919 & 1.014 & 1.109 & 1.251 \\
\hline 8 & 0.396 & 0.538 & 0.633 & 0.728 & 0.870 & 46 & 0.775 & 0.918 & 1.013 & 1.108 & 1.250 \\
\hline 9 & 0.447 & 0.589 & 0.684 & 0.779 & 0.922 & 47 & 0.775 & 0.917 & 1.012 & 1.107 & 1.250 \\
\hline 10 & 0.494 & 0.637 & 0.732 & 0.827 & 0.969 & 48 & 0.774 & 0.916 & 1.011 & 1.106 & 1.249 \\
\hline II & 0.538 & 0.681 & 0.776 & 0.871 & 1.013 & 49 & 0.773 & 0.916 & 1.011 & 1.106 & 1.248 \\
\hline 12 & 0.579 & 0.722 & 0.817 & 0.911 & 1.054 & 50 & 0.772 & 0.915 & 1.010 & 1.105 & 1.247 \\
\hline 13 & 0.616 & 0.759 & 0.854 & 0.949 & 1.091 & 51 & 0.772 & 0.914 & 1.009 & 1.104 & 1.247 \\
\hline 14 & 0.650 & 0.792 & 0.887 & 0.982 & 1.125 & 52 & $0.77 \mid$ & 0.913 & 1.008 & 1.103 & 1.246 \\
\hline 15 & 0.680 & 0.823 & 0.918 & 1.013 & 1.155 & 53 & 0.770 & 0.913 & 1.008 & 1.102 & 1.245 \\
\hline 16 & 0.707 & 0.849 & 0.944 & 1.039 & 1.182 & 54 & 0.769 & 0.912 & 1.007 & 1.102 & 1.244 \\
\hline 17 & 0.730 & 0.873 & 0.968 & 1.063 & 1.205 & 55 & 0.768 & 0.911 & 1.006 & 1.101 & 1.243 \\
\hline 18 & 0.750 & 0.892 & 0.987 & 1.082 & 1.225 & 56 & 0.768 & 0.910 & 1.005 & 1.100 & 1.243 \\
\hline 19 & 0.766 & 0.909 & 1.004 & 1.099 & 1.241 & 57 & 0.767 & 0.909 & 1.004 & 1.099 & 1.242 \\
\hline 20 & 0.779 & 0.922 & 1.017 & 1.112 & 1.254 & 58 & 0.766 & 0.908 & 1.003 & 1.098 & 1.241 \\
\hline 21 & 0.789 & 0.931 & 1.026 & 1.121 & 1.264 & 59 & 0.765 & 0.907 & 1.002 & 1.097 & 1.240 \\
\hline 22 & 0.796 & 0.938 & 1.033 & 1.128 & 1.271 & 60 & 0.764 & 0.906 & 1.001 & 1.096 & 1.239 \\
\hline 23 & 0.801 & 0.943 & 1.038 & 1.133 & 1.275 & 61 & 0.763 & 0.905 & 1.000 & 1.095 & 1.238 \\
\hline 24 & 0.803 & 0.946 & 1.041 & 1.136 & 1.278 & 62 & 0.762 & 0.904 & 0.999 & 1.094 & 1.236 \\
\hline 25 & 0.804 & 0.946 & I.04I & 1.136 & 1.279 & 63 & 0.760 & 0.903 & 0.998 & 1.093 & 1.235 \\
\hline 26 & 0.804 & 0.946 & 1.041 & 1.136 & 1.279 & 64 & 0.759 & 0.901 & 0.996 & 1.091 & 1.234 \\
\hline 27 & 0.802 & 0.945 & 1.040 & 1.135 & 1.277 & 65 & 0.758 & 0.900 & 0.995 & 1.090 & 1.232 \\
\hline 28 & 0.800 & 0.943 & 1.038 & 1.133 & 1.275 & 66 & 0.756 & 0.898 & 0.993 & 1.088 & 1.231 \\
\hline 29 & 0.798 & $0.94 I$ & 1.036 & 1.131 & 1.273 & 67 & 0.754 & 0.897 & 0.991 & 1.086 & 1.229 \\
\hline 30 & 0.796 & 0.939 & 1.034 & 1.129 & 1.271 & 68 & 0.752 & 0.894 & 0.989 & 1.084 & 1.227 \\
\hline 31 & 0.794 & 0.937 & 1.032 & 1.127 & 1.269 & 69 & 0.749 & 0.891 & 0.986 & 1.081 & 1.224 \\
\hline 32 & 0.792 & 0.935 & 1.030 & 1.125 & 1.267 & 70 & 0.746 & 0.888 & 0.983 & 1.078 & 1.220 \\
\hline 33 & $0.79 \mid$ & 0.933 & 1.028 & 1.123 & 1.265 & 71 & $0.74 I$ & 0.884 & 0.979 & 1.074 & 1.216 \\
\hline 34 & 0.789 & 0.931 & 1.026 & 1.121 & 1.264 & 72 & 0.736 & 0.879 & 0.974 & 1.069 & 1.211 \\
\hline 35 & 0.787 & 0.930 & 1.025 & 1.120 & 1.262 & 73 & $0.73 \mid$ & 0.873 & 0.968 & 1.063 & 1.206 \\
\hline 36 & 0.786 & 0.928 & 1.023 & 1.118 & 1.261 & 74 & 0.724 & 0.866 & 0.961 & 1.056 & 1.199 \\
\hline 37 & 0.785 & 0.927 & 1.022 & 1.117 & 1.259 & 75 & 0.716 & 0.858 & 0.953 & 1.048 & 1.191 \\
\hline 38 & 0.783 & 0.926 & 1.021 & 1.116 & 1.258 & 76 & 0.707 & 0.849 & 0.944 & 1.039 & 1.181 \\
\hline 39 & 0.782 & 0.925 & 1.020 & 1.115 & 1.257 & 77 & 0.696 & 0.839 & 0.934 & 1.028 & 1.171 \\
\hline 40 & $0.78 \mid$ & 0.924 & 1.019 & 1.113 & 1.256 & 78 & 0.684 & 0.827 & 0.922 & 1.016 & 1.159 \\
\hline 41 & 0.780 & 0.922 & 1.017 & 1.112 & 1.255 & 79 & $0.67 \mid$ & 0.813 & 0.908 & 1.003 & 1.145 \\
\hline 42 & 0.779 & 0.921 & 1.016 & 1.111 & 1.254 & 80 & 0.655 & 0.798 & 0.893 & 0.988 & 1.130 \\
\hline 43 & 0.778 & 0.921 & 1.016 & 1.110 & 1.253 & & & & & & \\
\hline 44 & 0.777 & 0.920 & 1.015 & 1.110 & 1.252 & & & & & & \\
\hline
\end{tabular}


Table IV.8

Bone Mineral Density Z score values of ribs for male population

Z SCORE

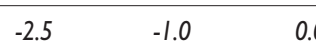

Age

P.

$-1.0$

0.447

0.522

0.554

0.585

0.614

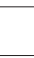

10

13

\begin{tabular}{llllll}
\hline 14 & 0.521 & 0.632 & 0.707 & 0.781 & 2.290 \\
\hline 15 & 0.539 & 0.650 & 0.725 & 0.799 & 2.374 \\
\hline
\end{tabular}

$\begin{array}{llllll}16 & 0.554 & 0.666 & 0.741 & 0.815 & 2.450\end{array}$

\begin{tabular}{llllll}
\hline 17 & 0.568 & 0.680 & 0.754 & 0.829 & 2.519
\end{tabular}

$\begin{array}{llllll}18 & 0.580 & 0.692 & 0.766 & 0.840 & 2.581 \\ 19 & 0.589 & 0.701 & 0.775 & 0.850 & 2.635\end{array}$$$
20
$$

\begin{tabular}{llllll}
20 & 0.597 & 0.708 & 0.783 & 0.857 & 2.683 \\
\hline 21 & 0.602 & 0.714 & 0.788 & 0.863 & 2.723 \\
\hline 22 & 0.606 & 0.718 & 0.792 & 0.867 & 2.756
\end{tabular}

\begin{tabular}{llllll}
22 & 0.606 & 0.718 & 0.792 & 0.867 & 2.756 \\
\hline 23 & 0.609 & 0.720 & 0.795 & 0.869 & 2.782 \\
\hline
\end{tabular}

\begin{tabular}{llllll}
\hline 24 & 0.610 & 0.721 & 0.796 & 0.870 & 2.802 \\
\hline 25 & 0.610 & 0.722 & 0.796 & 0.871 & 2.815 \\
\hline 26 & 0.609 & 0.721 & 0.796 & 0.870 & 2.824
\end{tabular}

\begin{tabular}{llllll}
\hline 26 & 0.609 & 0.721 & 0.796 & 0.870 & 2.824 \\
\hline 27 & 0.608 & 0.720 & 0.794 & 0.869 & 2.829
\end{tabular}

\begin{tabular}{llllll}
\hline 28 & 0.607 & 0.718 & 0.793 & 0.867 & 2.830 \\
\hline 29 & 0.605 & 0.717 & 0.791 & 0.866 & 2.829
\end{tabular}

\begin{tabular}{llllll}
\hline 30 & 0.603 & 0.715 & 0.790 & 0.864 & 2.826 \\
\hline
\end{tabular}

\begin{tabular}{llllll}
\hline 31 & 0.602 & 0.714 & 0.788 & 0.863 & 2.821 \\
\hline 32 & 0.601 & 0.712 & 0.787 & 0.861 & 2.816
\end{tabular}

\begin{tabular}{llllll}
34 & 0.598 & 0.710 & 0.784 & 0.859 & 2.806 \\
\hline 35 & 0.597 & 0.709 & 0.783 & 0.858 & 2.801
\end{tabular}

$\begin{array}{llllll}36 & 0.596 & 0.708 & 0.782 & 0.856 & 2.797\end{array}$

$\begin{array}{llllll}37 & 0.595 & 0.706 & 0.781 & 0.855 & 2.792\end{array}$

$\begin{array}{llllll}38 & 0.594 & 0.706 & 0.780 & 0.855 & 2.788 \\ 39 & 0.593 & 0.705 & 0.779 & 0.854 & 2.785\end{array}$

\begin{tabular}{llllll}
\hline 40 & 0.592 & 0.704 & 0.778 & 0.853 & 2.781 \\
\hline 41 & 0.591 & 0.703 & 0.778 & 0.852 & 2.778 \\
\hline 42 & 0.591 & 0.702 & 0.777 & 0.851 & 2.774 \\
\hline 43 & 0.590 & 0.702 & 0.776 & 0.851 & 2.771 \\
\hline 44 & 0.589 & 0.701 & 0.775 & 0.850 & 2.769
\end{tabular}

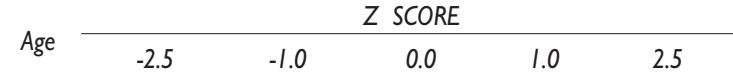

\begin{tabular}{llllll}
45 & 0.589 & 0.700 & 0.775 & 0.849 & 2.766 \\
\hline 46 & 0.588 & 0.700 & 0.774 & 0.849 & 2.763 \\
\hline
\end{tabular}

$\begin{array}{llllll}47 & 0.587 & 0.699 & 0.774 & 0.848 & 2.761\end{array}$

\begin{tabular}{llllll}
\hline 48 & 0.587 & 0.698 & 0.773 & 0.847 & 2.759 \\
\hline 49 & 0.586 & 0.698 & 0.772 & 0.847 & 2.757
\end{tabular}

\begin{tabular}{llllll}
\hline 50 & 0.586 & 0.697 & 0.772 & 0.846 & 2.754 \\
\hline 51 & 0.585 & 0.697 & 0.771 & 0.846 & 2.752 \\
\hline 52 & 0.585 & 0.696 & 0.771 & 0.845 & 2.750 \\
\hline
\end{tabular}

\begin{tabular}{llllll}
\hline 53 & 0.584 & 0.696 & 0.770 & 0.845 & 2.748 \\
\hline 54 & 0.583 & 0.695 & 0.770 & 0.844 & 2.747 \\
\hline 55 & 0.583 & 0.694 & 0.769 & 0.843 & 2.745 \\
\hline 56 & 0.582 & 0.694 & 0.768 & 0.843 & 2.743 \\
\hline
\end{tabular}

\begin{tabular}{llllll}
56 & 0.582 & 0.694 & 0.768 & 0.843 & 2.743 \\
\hline 57 & 0.581 & 0.693 & 0.768 & 0.842 & 2.741 \\
\hline 58 & 0.581 & 0.693 & 0.767 & 0.841 & 2.739 \\
\hline
\end{tabular}

\begin{tabular}{llllll}
\hline 59 & 0.580 & 0.692 & 0.766 & $0.84 I$ & 2.736 \\
\hline 60 & 0.579 & $0.69 I$ & 0.766 & 0.840 & 2.734
\end{tabular}

\begin{tabular}{llllll}
60 & 0.579 & 0.691 & 0.766 & 0.840 & 2.734 \\
\hline 61 & 0.579 & 0.690 & 0.765 & 0.839 & 2.732 \\
\hline 62 & 0.578 & 0.689 & 0.764 & 0.838 & 2.729 \\
\hline
\end{tabular}

\begin{tabular}{llllll}
\hline 63 & 0.577 & 0.689 & 0.763 & 0.837 & 2.726 \\
\hline 64 & 0.576 & 0.688 & 0.762 & 0.837 & 2.724 \\
\hline 65 & 0.575 & 0.687 & 0.761 & 0.836 & 2.721 \\
\hline
\end{tabular}

\begin{tabular}{|c|c|c|c|c|c|}
\hline & & & & & \\
\hline 66 & 0.574 & 0.685 & 0.760 & 0.834 & 2.717 \\
\hline 67 & 0.572 & 0.684 & 0.759 & 0.833 & $2.7 \mid 4$ \\
\hline 68 & 0.571 & 0.683 & 0.757 & 0.832 & 2.710 \\
\hline 69 & 0.569 & 0.681 & 0.755 & 0.830 & 2.706 \\
\hline 70 & 0.567 & 0.679 & 0.753 & 0.828 & 2.701 \\
\hline 71 & 0.565 & 0.677 & 0.751 & 0.826 & 2.696 \\
\hline 72 & 0.562 & 0.674 & 0.748 & 0.823 & 2.689 \\
\hline 73 & 0.559 & 0.671 & 0.745 & 0.820 & 2.682 \\
\hline 74 & 0.556 & 0.667 & 0.742 & 0.816 & 2.674 \\
\hline 75 & 0.552 & 0.663 & 0.738 & 0.812 & 2.664 \\
\hline 76 & 0.547 & 0.659 & 0.733 & 0.808 & 2.653 \\
\hline 77 & 0.542 & 0.654 & 0.728 & 0.802 & 2.640 \\
\hline 78 & 0.536 & 0.648 & 0.722 & 0.797 & 2.625 \\
\hline 79 & 0.529 & 0.641 & 0.716 & 0.790 & 2.609 \\
\hline 80 & 0.522 & 0.634 & 0.708 & 0.783 & 2.590 \\
\hline
\end{tabular}


Table IV.9

Bone Mineral Density Z score values of pelvis for male population

\begin{tabular}{|c|c|c|c|c|c|}
\hline \multirow{2}{*}{ Age } & \multicolumn{5}{|c|}{ Z SCORE } \\
\hline & -2.5 & -1.0 & 0.0 & 1.0 & 2.5 \\
\hline 7 & 0.318 & 0.520 & 0.654 & 0.789 & 0.991 \\
\hline 8 & 0.394 & 0.595 & 0.730 & 0.864 & 1.066 \\
\hline 9 & 0.464 & 0.666 & 0.800 & 0.934 & 1.136 \\
\hline 10 & 0.529 & 0.731 & 0.865 & 1.000 & 1.202 \\
\hline II & 0.590 & 0.791 & 0.926 & 1.060 & 1.262 \\
\hline 12 & 0.645 & 0.847 & 0.981 & 1.116 & 1.317 \\
\hline 13 & 0.696 & 0.897 & 1.032 & 1.166 & 1.368 \\
\hline 14 & $0.74 I$ & 0.943 & 1.077 & 1.212 & 1.414 \\
\hline 15 & 0.782 & 0.984 & 1.118 & 1.253 & 1.454 \\
\hline 16 & 0.818 & 1.019 & 1.154 & 1.288 & 1.490 \\
\hline 17 & 0.849 & 1.050 & 1.185 & 1.319 & 1.521 \\
\hline 18 & 0.874 & 1.076 & 1.211 & 1.345 & 1.547 \\
\hline 19 & 0.895 & 1.097 & 1.231 & 1.366 & 1.568 \\
\hline 20 & 0.911 & 1.113 & 1.247 & 1.382 & 1.584 \\
\hline 21 & 0.923 & 1.125 & 1.259 & 1.394 & 1.595 \\
\hline 22 & 0.931 & 1.132 & 1.267 & 1.401 & 1.603 \\
\hline 23 & 0.935 & 1.137 & 1.271 & 1.406 & 1.607 \\
\hline 24 & 0.937 & 1.138 & 1.273 & 1.407 & 1.609 \\
\hline 25 & 0.936 & 1.138 & 1.272 & I.407 & 1.608 \\
\hline 26 & 0.933 & 1.135 & 1.270 & 1.404 & 1.606 \\
\hline 27 & 0.930 & 1.131 & 1.266 & 1.400 & 1.602 \\
\hline 28 & 0.925 & 1.127 & 1.261 & 1.396 & 1.597 \\
\hline 29 & 0.921 & 1.122 & 1.257 & 1.391 & 1.593 \\
\hline 30 & 0.916 & 1.118 & 1.252 & 1.387 & 1.588 \\
\hline 31 & 0.912 & 1.114 & 1.248 & 1.383 & 1.584 \\
\hline 32 & 0.908 & 1.110 & 1.244 & 1.379 & 1.580 \\
\hline 33 & 0.905 & 1.106 & $1.24 \mid$ & 1.375 & 1.577 \\
\hline 34 & 0.901 & 1.103 & 1.237 & 1.372 & 1.573 \\
\hline 35 & 0.898 & 1.100 & 1.234 & 1.369 & 1.570 \\
\hline 36 & 0.895 & 1.097 & 1.231 & 1.366 & 1.568 \\
\hline 37 & 0.893 & 1.094 & 1.229 & 1.363 & 1.565 \\
\hline 38 & 0.890 & 1.092 & 1.226 & 1.361 & 1.563 \\
\hline 39 & 0.888 & 1.090 & 1.224 & 1.359 & 1.560 \\
\hline 40 & 0.886 & 1.088 & 1.222 & 1.356 & 1.558 \\
\hline 41 & 0.884 & 1.086 & 1.220 & 1.354 & 1.556 \\
\hline 42 & 0.882 & 1.084 & 1.218 & 1.353 & 1.554 \\
\hline 43 & 0.880 & 1.082 & 1.216 & 1.351 & 1.553 \\
\hline 44 & 0.879 & 1.080 & 1.215 & 1.349 & $|.55|$ \\
\hline
\end{tabular}

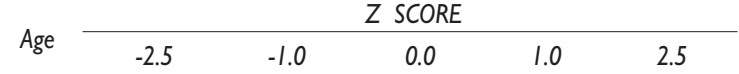

\begin{tabular}{|c|c|c|c|c|c|}
\hline 45 & 0.877 & 1.079 & 1.213 & 1.348 & 1.549 \\
\hline 46 & 0.875 & 1.077 & 1.212 & 1.346 & 1.548 \\
\hline 47 & 0.874 & 1.076 & 1.210 & 1.344 & 1.546 \\
\hline 48 & 0.872 & 1.074 & 1.209 & 1.343 & 1.545 \\
\hline 49 & 0.871 & 1.073 & 1.207 & 1.341 & 1.543 \\
\hline 50 & 0.869 & $1.07 \mid$ & 1.205 & 1.340 & 1.542 \\
\hline 51 & 0.868 & 1.069 & 1.204 & 1.338 & 1.540 \\
\hline 52 & 0.866 & 1.068 & 1.202 & 1.337 & 1.538 \\
\hline 53 & 0.864 & 1.066 & 1.200 & 1.335 & 1.537 \\
\hline 54 & 0.863 & 1.064 & 1.199 & 1.333 & 1.535 \\
\hline 55 & 0.861 & 1.062 & I.197 & 1.331 & 1.533 \\
\hline 56 & 0.859 & 1.060 & 1.195 & 1.329 & $|.53|$ \\
\hline 57 & 0.856 & 1.058 & 1.193 & 1.327 & 1.529 \\
\hline 58 & 0.854 & 1.056 & 1.190 & 1.325 & 1.526 \\
\hline 59 & 0.852 & 1.053 & 1.188 & 1.322 & 1.524 \\
\hline 60 & 0.849 & 1.050 & 1.185 & 1.319 & 1.521 \\
\hline 61 & 0.846 & 1.048 & 1.182 & 1.316 & 1.518 \\
\hline 62 & 0.843 & 1.044 & I. 179 & 1.313 & 1.515 \\
\hline 63 & 0.839 & 1.041 & I.I75 & 1.310 & 1.511 \\
\hline 64 & 0.836 & 1.037 & I. 172 & 1.306 & 1.508 \\
\hline 65 & 0.832 & 1.033 & 1.168 & 1.302 & 1.504 \\
\hline 66 & 0.827 & 1.029 & 1.163 & 1.298 & 1.499 \\
\hline 67 & 0.822 & 1.024 & 1.159 & 1.293 & I.495 \\
\hline 68 & 0.817 & 1.019 & 1.153 & 1.288 & 1.489 \\
\hline 69 & 0.811 & 1.013 & I. 147 & 1.282 & I.483 \\
\hline 70 & 0.804 & 1.006 & 1.141 & 1.275 & I.477 \\
\hline 71 & 0.797 & 0.999 & 1.133 & 1.268 & 1.469 \\
\hline 72 & 0.789 & 0.990 & 1.125 & 1.259 & $\mathrm{I} .46 \mathrm{I}$ \\
\hline 73 & 0.780 & 0.981 & 1.116 & 1.250 & 1.452 \\
\hline 74 & 0.769 & 0.971 & 1.105 & 1.240 & I.44I \\
\hline 75 & 0.758 & 0.960 & 1.094 & 1.228 & 1.430 \\
\hline 76 & 0.745 & 0.947 & 1.081 & 1.216 & 1.418 \\
\hline 77 & 0.732 & 0.933 & 1.068 & 1.202 & I.404 \\
\hline 78 & 0.716 & 0.918 & 1.052 & 1.187 & 1.389 \\
\hline 79 & 0.700 & 0.901 & 1.036 & 1.170 & 1.372 \\
\hline 80 & 0.682 & 0.883 & 1.018 & 1.152 & 1.354 \\
\hline
\end{tabular}


Table IV. 10

Bone Mineral Density Z score values of subtotal body for female population

Age

\begin{tabular}{|c|c|c|c|c|c|}
\hline 7 & 0.622 & $0.74 I$ & 0.820 & 0.899 & 1.018 \\
\hline 8 & 0.661 & 0.780 & 0.859 & 0.938 & 1.057 \\
\hline 9 & 0.697 & 0.816 & 0.895 & 0.975 & 1.093 \\
\hline 10 & 0.732 & 0.850 & 0.930 & 1.009 & 1.128 \\
\hline II & 0.764 & 0.882 & 0.962 & 1.041 & 1.160 \\
\hline 12 & 0.793 & 0.912 & 0.991 & I.07I & 1.189 \\
\hline 13 & 0.821 & 0.940 & 1.019 & 1.098 & 1.217 \\
\hline 14 & 0.846 & 0.965 & 1.044 & 1.123 & 1.242 \\
\hline 15 & 0.869 & 0.988 & 1.067 & I.I46 & 1.265 \\
\hline 16 & 0.889 & 1.008 & 1.087 & 1.167 & 1.285 \\
\hline 17 & 0.907 & 1.026 & 1.106 & 1.185 & 1.304 \\
\hline 18 & 0.923 & 1.042 & 1.121 & 1.201 & 1.320 \\
\hline 19 & 0.937 & 1.056 & 1.135 & 1.214 & 1.333 \\
\hline 20 & 0.948 & 1.067 & 1.147 & 1.226 & 1.345 \\
\hline 21 & 0.958 & 1.077 & 1.156 & 1.235 & 1.354 \\
\hline 22 & 0.965 & 1.084 & 1.163 & 1.242 & 1.361 \\
\hline 23 & 0.971 & 1.090 & 1.169 & 1.248 & 1.367 \\
\hline 24 & 0.975 & 1.094 & 1.173 & 1.252 & I.37| \\
\hline 25 & 0.978 & 1.097 & 1.176 & 1.255 & 1.374 \\
\hline 26 & 0.980 & 1.099 & 1.178 & 1.257 & 1.376 \\
\hline 27 & 0.981 & 1.100 & 1.179 & 1.258 & 1.377 \\
\hline 28 & 0.982 & 1.100 & 1.180 & 1.259 & 1.378 \\
\hline 29 & 0.982 & 1.100 & 1.180 & 1.259 & 1.378 \\
\hline 30 & 0.981 & 1.100 & 1.179 & 1.259 & 1.377 \\
\hline 31 & 0.981 & 1.100 & 1.179 & 1.258 & 1.377 \\
\hline 32 & 0.980 & 1.099 & 1.178 & 1.257 & 1.376 \\
\hline 33 & 0.979 & 1.098 & 1.177 & 1.256 & 1.375 \\
\hline 34 & 0.978 & 1.096 & 1.176 & 1.255 & 1.374 \\
\hline 35 & 0.976 & 1.095 & 1.174 & 1.253 & 1.372 \\
\hline 36 & 0.974 & 1.093 & 1.172 & 1.252 & 1.370 \\
\hline 37 & 0.972 & 1.091 & 1.170 & 1.250 & 1.369 \\
\hline 38 & 0.970 & 1.089 & 1.168 & I.247 & 1.366 \\
\hline 39 & 0.968 & 1.087 & 1.166 & 1.245 & 1.364 \\
\hline 40 & 0.965 & 1.084 & 1.163 & 1.242 & 1.361 \\
\hline 41 & 0.962 & 1.081 & 1.160 & 1.240 & 1.358 \\
\hline 42 & 0.959 & 1.078 & 1.157 & 1.236 & 1.355 \\
\hline 43 & 0.956 & 1.075 & 1.154 & 1.233 & 1.352 \\
\hline 44 & 0.952 & I.07| & 1.150 & 1.230 & 1.349 \\
\hline
\end{tabular}

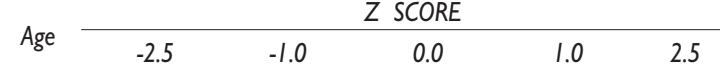

\begin{tabular}{|c|c|c|c|c|c|}
\hline 45 & 0.949 & 1.067 & I.I47 & 1.226 & 1.345 \\
\hline 46 & 0.945 & 1.064 & 1.143 & 1.222 & I.34I \\
\hline 47 & $0.94 I$ & 1.059 & 1.139 & 1.218 & 1.337 \\
\hline 48 & 0.936 & 1.055 & 1.134 & 1.214 & 1.332 \\
\hline 49 & 0.932 & 1.051 & 1.130 & 1.209 & 1.328 \\
\hline 50 & 0.927 & 1.046 & 1.125 & 1.204 & 1.323 \\
\hline 51 & 0.922 & 1.041 & 1.120 & 1.199 & 1.318 \\
\hline 52 & 0.917 & 1.036 & 1.115 & 1.194 & 1.313 \\
\hline 53 & 0.911 & 1.030 & 1.110 & 1.189 & 1.308 \\
\hline 54 & 0.906 & 1.025 & 1.104 & 1.183 & 1.302 \\
\hline 55 & 0.900 & 1.019 & 1.098 & 1.178 & 1.296 \\
\hline 56 & 0.894 & 1.013 & 1.092 & 1.172 & 1.291 \\
\hline 57 & 0.888 & 1.007 & 1.086 & 1.166 & 1.284 \\
\hline 58 & 0.882 & 1.001 & 1.080 & 1.159 & 1.278 \\
\hline 59 & 0.875 & 0.994 & 1.074 & 1.153 & 1.272 \\
\hline 60 & 0.869 & 0.988 & 1.067 & 1.146 & 1.265 \\
\hline 61 & 0.862 & 0.981 & 1.060 & 1.139 & 1.258 \\
\hline 62 & 0.855 & 0.974 & 1.053 & 1.132 & 1.251 \\
\hline 63 & 0.848 & 0.967 & 1.046 & 1.125 & 1.244 \\
\hline 64 & 0.841 & 0.959 & 1.039 & 1.118 & 1.237 \\
\hline 65 & 0.833 & 0.952 & 1.031 & 1.110 & 1.229 \\
\hline 66 & 0.826 & 0.944 & 1.024 & 1.103 & 1.222 \\
\hline 67 & 0.818 & 0.937 & 1.016 & 1.095 & 1.214 \\
\hline 68 & 0.811 & 0.929 & 1.009 & 1.088 & 1.207 \\
\hline 69 & 0.803 & 0.922 & 1.001 & 1.081 & 1.200 \\
\hline 70 & 0.796 & 0.915 & 0.994 & 1.074 & 1.193 \\
\hline 71 & 0.790 & 0.909 & 0.988 & 1.067 & 1.186 \\
\hline 72 & 0.784 & 0.903 & 0.982 & 1.061 & 1.180 \\
\hline 73 & 0.778 & 0.897 & 0.976 & 1.056 & I.175 \\
\hline 74 & 0.774 & 0.892 & 0.972 & 1.051 & 1.170 \\
\hline 75 & 0.770 & 0.888 & 0.968 & 1.047 & 1.166 \\
\hline 76 & 0.766 & 0.885 & 0.964 & 1.044 & 1.163 \\
\hline 77 & 0.764 & 0.883 & 0.962 & I.04I & 1.160 \\
\hline 78 & 0.763 & 0.882 & 0.961 & 1.040 & 1.159 \\
\hline 79 & 0.761 & 0.880 & 0.959 & 1.038 & 1.157 \\
\hline 80 & 0.759 & 0.878 & 0.957 & 1.036 & 1.155 \\
\hline
\end{tabular}


Table IV.II

Bone Mineral Density Z score values of lumbar region for female population

\begin{tabular}{|c|c|c|c|c|c|}
\hline \multirow{2}{*}{ Age } & \multicolumn{5}{|c|}{ Z SCORE } \\
\hline & -2.5 & -1.0 & 0.0 & 1.0 & 2.5 \\
\hline 7 & 0.315 & 0.517 & 0.652 & 0.787 & 0.988 \\
\hline 8 & 0.368 & 0.570 & 0.705 & 0.839 & I.04I \\
\hline 9 & 0.418 & 0.620 & 0.755 & 0.889 & 1.091 \\
\hline 10 & 0.465 & 0.667 & 0.802 & 0.936 & 1.138 \\
\hline 11 & 0.509 & 0.711 & 0.846 & 0.980 & 1.182 \\
\hline 12 & 0.550 & 0.752 & 0.887 & 1.021 & 1.223 \\
\hline 13 & 0.589 & 0.791 & 0.925 & 1.060 & 1.262 \\
\hline 14 & 0.624 & 0.826 & 0.961 & 1.095 & I.297 \\
\hline 15 & 0.657 & 0.859 & 0.993 & 1.128 & 1.330 \\
\hline 16 & 0.686 & 0.888 & 1.023 & 1.158 & 1.359 \\
\hline 17 & 0.713 & 0.915 & 1.050 & I.184 & 1.386 \\
\hline 18 & 0.737 & 0.939 & 1.074 & 1.208 & 1.410 \\
\hline 19 & 0.758 & 0.960 & 1.095 & 1.229 & 1.431 \\
\hline 20 & 0.776 & 0.978 & 1.113 & 1.247 & 1.449 \\
\hline 21 & 0.792 & 0.994 & I.I28 & 1.263 & 1.465 \\
\hline 22 & 0.805 & 1.007 & I.I4I & 1.276 & I.478 \\
\hline 23 & 0.816 & 1.018 & I.I52 & 1.287 & 1.489 \\
\hline 24 & 0.825 & 1.026 & 1.161 & 1.296 & 1.498 \\
\hline 25 & 0.831 & 1.033 & 1.168 & 1.303 & 1.504 \\
\hline 26 & 0.837 & 1.039 & I.173 & 1.308 & 1.510 \\
\hline 27 & $0.84 I$ & 1.042 & I.I77 & 1.312 & 1.514 \\
\hline 28 & 0.843 & 1.045 & I. 180 & 1.314 & 1.516 \\
\hline 29 & 0.845 & 1.047 & 1.181 & 1.316 & 1.518 \\
\hline 30 & 0.845 & 1.047 & 1.182 & 1.317 & 1.519 \\
\hline 31 & 0.845 & 1.047 & 1.182 & 1.317 & 1.518 \\
\hline 32 & 0.845 & 1.047 & I.I8I & 1.316 & 1.518 \\
\hline 33 & 0.843 & 1.045 & I. 180 & 1.314 & 1.516 \\
\hline 34 & $0.84 I$ & 1.043 & 1.178 & 1.312 & 1.514 \\
\hline 35 & 0.838 & 1.040 & I.I75 & 1.309 & 1.511 \\
\hline 36 & 0.835 & 1.037 & I.I7I & 1.306 & 1.508 \\
\hline 37 & 0.831 & 1.033 & 1.167 & 1.302 & 1.504 \\
\hline 38 & 0.826 & 1.028 & 1.163 & 1.297 & 1.499 \\
\hline 39 & 0.821 & 1.023 & I.I58 & 1.292 & 1.494 \\
\hline 40 & 0.816 & 1.018 & 1.152 & 1.287 & 1.489 \\
\hline 41 & 0.810 & 1.012 & 1.146 & 1.281 & 1.483 \\
\hline 42 & 0.803 & 1.005 & 1.140 & 1.274 & 1.476 \\
\hline 43 & 0.796 & 0.998 & 1.133 & 1.268 & 1.469 \\
\hline 44 & 0.789 & 0.991 & 1.126 & 1.260 & 1.462 \\
\hline
\end{tabular}

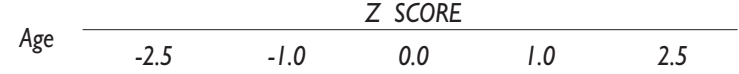

\begin{tabular}{llllll}
45 & 0.782 & 0.984 & 1.118 & 1.253 & 1.455 \\
\hline 46 & 0.774 & 0.976 & 1.111 & 1.245 & 1.447 \\
\hline
\end{tabular}

$\begin{array}{llllll}47 & 0.766 & 0.968 & 1.102 & 1.237 & 1.439\end{array}$

\begin{tabular}{llllll}
\hline 48 & 0.758 & 0.960 & 1.094 & 1.229 & 1.431 \\
\hline 49 & 0.749 & 0.951 & 1.086 & 1.220 & 1.422
\end{tabular}

\begin{tabular}{llllll}
\hline 50 & 0.741 & 0.942 & 1.077 & 1.212 & 1.414 \\
\hline 51 & 0.732 & 0.934 & 1.068 & 1.203 & 1.405 \\
\hline 52 & 0.723 & 0.925 & 1.059 & 1.194 & 1.396
\end{tabular}

\begin{tabular}{llllll}
\hline 53 & 0.714 & 0.916 & 1.051 & 1.185 & 1.387 \\
\hline 54 & 0.705 & 0.907 & 1.042 & 1.176 & 1.378
\end{tabular}

\begin{tabular}{llllll}
\hline 54 & 0.705 & 0.907 & 1.042 & 1.176 & 1.378 \\
\hline 55 & 0.696 & 0.898 & 1.033 & 1.167 & 1.369
\end{tabular}

\begin{tabular}{llllll}
\hline 56 & 0.687 & 0.889 & 1.024 & 1.158 & 1.360 \\
\hline 57 & 0.678 & 0.880 & 1.015 & 1.149 & 1.351
\end{tabular}

\begin{tabular}{llllll}
57 & 0.678 & 0.880 & 1.015 & 1.149 & 1.351 \\
\hline 58 & 0.670 & 0.871 & 1.006 & 1.141 & 1.343 \\
\hline
\end{tabular}

\begin{tabular}{llllll}
\hline 59 & 0.661 & 0.863 & 0.997 & 1.132 & 1.334 \\
\hline 60 & 0.652 & 0.854 & 0.989 & 1.124 & 1.325
\end{tabular}

\begin{tabular}{llllll}
\hline 61 & 0.644 & 0.846 & 0.981 & 1.115 & 1.317 \\
\hline 62 & 0.636 & 0.838 & 0.973 & 1.107 & 1.309
\end{tabular}

\begin{tabular}{llllll}
\hline 63 & 0.628 & 0.830 & 0.965 & 1.099 & $\mathrm{I} .30 \mathrm{I}$ \\
\hline 64 & 0.621 & 0.823 & 0.957 & 1.092 & $\mathrm{I} .294$
\end{tabular}

\begin{tabular}{llllll}
\hline 65 & 0.614 & 0.815 & 0.950 & 1.085 & 1.287 \\
\hline
\end{tabular}

\begin{tabular}{rllllll}
\hline 66 & 0.607 & 0.809 & 0.943 & 1.078 & 1.280 \\
\hline 67 & 0.600 & 0.802 & 0.936 & $1.07 \mathrm{I}$ & $\mathrm{I} .273$
\end{tabular}

\begin{tabular}{llllll}
\hline 68 & 0.594 & 0.795 & 0.930 & 1.065 & 1.267 \\
\hline 69 & 0.587 & 0.789 & 0.924 & 1.058 & 1.260
\end{tabular}

\begin{tabular}{llllll}
\hline 70 & 0.581 & 0.783 & 0.918 & 1.052 & 1.254 \\
\hline 71 & 0.575 & 0.777 & 0.912 & 1.046 & 1.248 \\
\hline
\end{tabular}

\begin{tabular}{llllll}
\hline 72 & 0.570 & 0.771 & 0.906 & 1.041 & 1.243 \\
\hline 73 & 0.564 & 0.766 & 0.900 & 1.035 & 1.237
\end{tabular}

\begin{tabular}{llllll}
\hline 73 & 0.564 & 0.766 & 0.900 & 1.035 & 1.237 \\
\hline 74 & 0.558 & 0.760 & 0.895 & 1.029 & $\mathrm{I} .23 \mathrm{I}$ \\
\hline 75 & 0.553 & 0.755 & 0.889 & 1.024 & $\mathrm{I} .226$ \\
\hline 76 & 0.547 & 0.749 & 0.884 & 1.018 & $\mathrm{I} .220$ \\
\hline 77 & $0.54 \mathrm{I}$ & 0.743 & 0.878 & $\mathrm{I} .012$ & $\mathrm{I} .2 \mathrm{I} 4$ \\
\hline 78 & 0.536 & 0.738 & 0.872 & $\mathrm{I} .007$ & $\mathrm{I} .209$ \\
\hline 79 & 0.530 & 0.732 & 0.866 & $\mathrm{I} .00 \mathrm{I}$ & $\mathrm{I} .203$ \\
\hline 80 & 0.524 & 0.726 & 0.860 & 0.995 & $\mathrm{I} .197$
\end{tabular}


Table IV. 12

Bone Mineral Density Z score values of hip for female population

Age

\begin{tabular}{|c|c|c|c|c|c|}
\hline 7 & 0.444 & 0.623 & 0.743 & 0.863 & 1.042 \\
\hline 8 & 0.483 & 0.662 & 0.782 & 0.902 & 1.081 \\
\hline 9 & 0.519 & 0.699 & 0.819 & 0.938 & 1.118 \\
\hline 10 & 0.554 & 0.733 & 0.853 & 0.972 & I.152 \\
\hline II & 0.585 & 0.765 & 0.884 & 1.004 & 1.183 \\
\hline 12 & 0.614 & 0.793 & 0.913 & 1.033 & 1.212 \\
\hline 13 & 0.640 & 0.820 & 0.940 & 1.059 & 1.239 \\
\hline 14 & 0.664 & 0.844 & 0.964 & 1.083 & 1.263 \\
\hline 15 & 0.686 & 0.865 & 0.985 & 1.105 & 1.284 \\
\hline 16 & 0.705 & 0.884 & 1.004 & 1.124 & 1.303 \\
\hline 17 & 0.721 & 0.901 & 1.020 & 1.140 & 1.319 \\
\hline 18 & 0.735 & 0.914 & 1.034 & 1.154 & 1.333 \\
\hline 19 & 0.746 & 0.926 & 1.045 & 1.165 & 1.345 \\
\hline 20 & 0.755 & 0.935 & 1.054 & I.I74 & 1.354 \\
\hline 21 & 0.762 & $0.94 I$ & 1.061 & 1.181 & 1.360 \\
\hline 22 & 0.766 & 0.946 & 1.066 & 1.185 & 1.365 \\
\hline 23 & 0.769 & 0.949 & 1.068 & 1.188 & 1.368 \\
\hline 24 & 0.770 & 0.950 & 1.070 & 1.189 & 1.369 \\
\hline 25 & 0.770 & 0.950 & 1.070 & I.189 & 1.369 \\
\hline 26 & 0.770 & 0.949 & 1.069 & 1.188 & 1.368 \\
\hline 27 & 0.768 & 0.947 & 1.067 & I.I87 & 1.366 \\
\hline 28 & 0.765 & 0.945 & 1.065 & I.184 & 1.364 \\
\hline 29 & 0.763 & 0.942 & 1.062 & 1.182 & 1.361 \\
\hline 30 & 0.760 & 0.940 & 1.060 & 1.179 & 1.359 \\
\hline 31 & 0.758 & 0.937 & 1.057 & I.I77 & 1.356 \\
\hline 32 & 0.755 & 0.935 & 1.054 & I. 174 & 1.354 \\
\hline 33 & 0.752 & 0.932 & 1.052 & I.I7I & 1.351 \\
\hline 34 & 0.750 & 0.929 & 1.049 & 1.169 & 1.348 \\
\hline 35 & 0.747 & 0.926 & 1.046 & 1.166 & 1.345 \\
\hline 36 & 0.744 & 0.924 & 1.043 & 1.163 & 1.342 \\
\hline 37 & $0.74 I$ & 0.921 & 1.040 & 1.160 & 1.339 \\
\hline 38 & 0.738 & 0.918 & 1.037 & I.I57 & 1.336 \\
\hline 39 & 0.735 & 0.914 & 1.034 & 1.154 & 1.333 \\
\hline 40 & 0.732 & 0.911 & 1.031 & 1.151 & 1.330 \\
\hline 41 & 0.728 & 0.908 & 1.028 & 1.147 & I.327 \\
\hline 42 & 0.725 & 0.905 & 1.024 & 1.144 & 1.323 \\
\hline 43 & 0.721 & 0.901 & 1.021 & 1.140 & 1.320 \\
\hline 44 & 0.718 & 0.897 & 1.017 & I.I37 & 1.316 \\
\hline
\end{tabular}

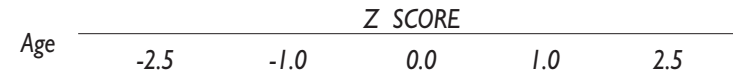

\begin{tabular}{|c|c|c|c|c|c|}
\hline 45 & 0.714 & 0.893 & 1.013 & 1.133 & 1.312 \\
\hline 46 & 0.710 & 0.889 & 1.009 & 1.129 & 1.308 \\
\hline 47 & 0.706 & 0.885 & 1.005 & 1.125 & 1.304 \\
\hline 48 & 0.701 & 0.881 & 1.001 & 1.120 & 1.300 \\
\hline 49 & 0.697 & 0.877 & 0.996 & 1.116 & 1.295 \\
\hline 50 & 0.692 & 0.872 & 0.992 & I.III & 1.291 \\
\hline 51 & 0.688 & 0.867 & 0.987 & 1.106 & 1.286 \\
\hline 52 & 0.682 & 0.862 & 0.982 & 1.101 & 1.281 \\
\hline 53 & 0.677 & 0.857 & 0.976 & 1.096 & 1.276 \\
\hline 54 & 0.672 & 0.851 & 0.971 & 1.091 & 1.270 \\
\hline 55 & 0.666 & 0.846 & 0.965 & 1.085 & 1.264 \\
\hline 56 & 0.660 & 0.840 & 0.959 & 1.079 & 1.259 \\
\hline 57 & 0.654 & 0.833 & 0.953 & 1.073 & 1.252 \\
\hline 58 & 0.648 & 0.827 & 0.947 & 1.066 & 1.246 \\
\hline 59 & $0.64 I$ & 0.820 & 0.940 & 1.060 & 1.239 \\
\hline 60 & 0.634 & 0.813 & 0.933 & 1.053 & 1.232 \\
\hline 61 & 0.627 & 0.806 & 0.926 & 1.046 & 1.225 \\
\hline 62 & 0.619 & 0.799 & 0.918 & 1.038 & 1.218 \\
\hline 63 & 0.611 & 0.791 & 0.911 & 1.030 & 1.210 \\
\hline 64 & 0.603 & 0.783 & 0.903 & 1.022 & 1.202 \\
\hline 65 & 0.595 & 0.774 & 0.894 & 1.014 & 1.193 \\
\hline 66 & 0.586 & 0.766 & 0.886 & 1.005 & 1.185 \\
\hline 67 & 0.578 & 0.757 & 0.877 & 0.997 & 1.176 \\
\hline 68 & 0.569 & 0.749 & 0.868 & 0.988 & 1.168 \\
\hline 69 & 0.561 & 0.740 & 0.860 & 0.980 & 1.159 \\
\hline 70 & 0.552 & 0.732 & 0.852 & 0.971 & 1.151 \\
\hline 71 & 0.545 & 0.724 & 0.844 & 0.963 & 1.143 \\
\hline 72 & 0.537 & 0.717 & 0.836 & 0.956 & 1.136 \\
\hline 73 & 0.530 & 0.710 & 0.829 & 0.949 & 1.129 \\
\hline 74 & 0.524 & 0.704 & 0.823 & 0.943 & 1.123 \\
\hline 75 & 0.519 & 0.698 & 0.818 & 0.938 & 1.117 \\
\hline 76 & 0.514 & 0.694 & 0.814 & 0.933 & 1.113 \\
\hline 77 & 0.511 & 0.691 & 0.810 & 0.930 & 1.109 \\
\hline 78 & 0.509 & 0.688 & 0.808 & 0.928 & I.107 \\
\hline 79 & 0.507 & 0.686 & 0.806 & 0.926 & 1.105 \\
\hline 80 & 0.505 & 0.684 & 0.804 & 0.924 & 1.103 \\
\hline
\end{tabular}


Table IV.I3

Bone Mineral Density Z score values of head for female population

\begin{tabular}{|c|c|c|c|c|c|}
\hline \multirow{2}{*}{ Age } & \multicolumn{5}{|c|}{ Z SCORE } \\
\hline & -2.5 & -1.0 & 0.0 & 1.0 & 2.5 \\
\hline 7 & 0.637 & 1.048 & 1.322 & 1.597 & 2.008 \\
\hline 8 & 0.744 & 1.156 & 1.430 & I.704 & 2.116 \\
\hline 9 & 0.846 & 1.258 & 1.532 & 1.806 & 2.217 \\
\hline 10 & 0.942 & 1.354 & 1.628 & 1.902 & 2.314 \\
\hline 11 & 1.033 & 1.444 & 1.719 & 1.993 & 2.404 \\
\hline 12 & 1.118 & 1.529 & 1.804 & 2.078 & 2.489 \\
\hline 13 & 1.197 & 1.608 & 1.883 & 2.157 & 2.568 \\
\hline 14 & 1.271 & 1.682 & 1.956 & 2.231 & 2.642 \\
\hline 15 & 1.339 & 1.750 & 2.024 & 2.298 & 2.710 \\
\hline 16 & 1.401 & 1.812 & 2.086 & 2.361 & 2.772 \\
\hline 17 & 1.457 & 1.869 & 2.143 & 2.417 & 2.828 \\
\hline 18 & 1.508 & 1.919 & 2.194 & 2.468 & 2.879 \\
\hline 19 & 1.553 & 1.965 & 2.239 & 2.513 & 2.925 \\
\hline 20 & 1.593 & 2.004 & 2.278 & 2.553 & 2.964 \\
\hline 21 & 1.627 & 2.039 & 2.313 & 2.587 & 2.998 \\
\hline 22 & 1.657 & 2.068 & 2.342 & 2.616 & 3.028 \\
\hline 23 & 1.682 & 2.093 & 2.367 & $2.64 I$ & 3.053 \\
\hline 24 & 1.703 & 2.114 & 2.388 & 2.662 & 3.074 \\
\hline 25 & 1.720 & 2.131 & 2.406 & 2.680 & 3.091 \\
\hline 26 & 1.734 & 2.145 & 2.420 & 2.694 & 3.105 \\
\hline 27 & 1.745 & 2.157 & 2.431 & 2.705 & 3.117 \\
\hline 28 & 1.754 & 2.166 & 2.440 & 2.714 & 3.125 \\
\hline 29 & 1.761 & 2.172 & 2.447 & 2.721 & 3.132 \\
\hline 30 & 1.766 & 2.178 & 2.452 & 2.726 & 3.138 \\
\hline 31 & 1.770 & 2.181 & 2.456 & 2.730 & 3.141 \\
\hline 32 & 1.772 & 2.184 & 2.458 & 2.732 & 3.144 \\
\hline 33 & 1.773 & 2.185 & 2.459 & 2.733 & 3.144 \\
\hline 34 & 1.773 & 2.184 & 2.458 & 2.733 & 3.144 \\
\hline 35 & 1.771 & 2.182 & 2.457 & 2.731 & 3.142 \\
\hline 36 & 1.768 & 2.179 & 2.454 & 2.728 & 3.139 \\
\hline 37 & 1.764 & 2.175 & 2.450 & 2.724 & 3.135 \\
\hline 38 & 1.759 & 2.170 & 2.444 & 2.719 & 3.130 \\
\hline 39 & 1.753 & 2.164 & 2.438 & 2.712 & 3.124 \\
\hline 40 & 1.745 & 2.157 & 2.431 & 2.705 & 3.117 \\
\hline 41 & 1.737 & 2.149 & 2.423 & 2.697 & 3.108 \\
\hline 42 & 1.728 & 2.140 & 2.414 & 2.688 & 3.099 \\
\hline 43 & 1.718 & 2.130 & 2.404 & 2.678 & 3.090 \\
\hline 44 & 1.708 & 2.119 & 2.394 & 2.668 & 3.079 \\
\hline
\end{tabular}

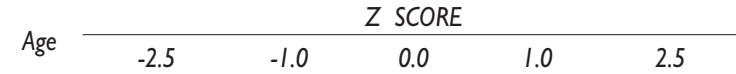

\begin{tabular}{|c|c|c|c|c|c|}
\hline 45 & 1.697 & 2.108 & 2.382 & 2.657 & 3.068 \\
\hline 46 & 1.685 & 2.096 & $2.37 \mid$ & 2.645 & 3.056 \\
\hline 47 & 1.673 & 2.084 & 2.358 & 2.633 & 3.044 \\
\hline 48 & 1.660 & $2.07 I$ & 2.346 & 2.620 & 3.031 \\
\hline 49 & 1.647 & 2.058 & 2.332 & 2.607 & 3.018 \\
\hline 50 & 1.633 & 2.045 & 2.319 & 2.593 & 3.005 \\
\hline 51 & 1.620 & 2.031 & 2.305 & 2.579 & 2.991 \\
\hline 52 & 1.605 & 2.017 & 2.291 & 2.565 & 2.977 \\
\hline 53 & 1.591 & 2.003 & 2.277 & 2.551 & 2.962 \\
\hline 54 & 1.577 & 1.988 & 2.263 & 2.537 & 2.948 \\
\hline 55 & 1.563 & 1.974 & 2.248 & 2.522 & 2.934 \\
\hline 56 & 1.548 & 1.960 & 2.234 & 2.508 & 2.919 \\
\hline 57 & 1.534 & 1.945 & 2.219 & 2.494 & 2.905 \\
\hline 58 & 1.520 & 1.931 & 2.205 & 2.480 & 2.891 \\
\hline 59 & 1.506 & 1.917 & 2.191 & 2.466 & 2.877 \\
\hline 60 & 1.492 & 1.903 & 2.178 & 2.452 & 2.863 \\
\hline 61 & 1.479 & 1.890 & 2.164 & 2.439 & 2.850 \\
\hline 62 & 1.466 & 1.877 & 2.151 & 2.426 & 2.837 \\
\hline 63 & 1.453 & 1.865 & 2.139 & 2.413 & 2.824 \\
\hline 64 & I.44I & 1.853 & 2.127 & 2.401 & 2.812 \\
\hline 65 & 1.430 & 1.841 & 2.115 & 2.390 & 2.801 \\
\hline 66 & 1.419 & 1.830 & 2.104 & 2.378 & 2.790 \\
\hline 67 & 1.408 & 1.819 & 2.093 & 2.368 & 2.779 \\
\hline 68 & 1.397 & 1.809 & 2.083 & 2.357 & 2.768 \\
\hline 69 & 1.387 & 1.798 & 2.072 & 2.346 & 2.758 \\
\hline 70 & 1.376 & I.787 & 2.061 & 2.336 & 2.747 \\
\hline 71 & 1.365 & 1.776 & 2.050 & 2.324 & 2.736 \\
\hline 72 & 1.353 & 1.764 & 2.039 & 2.313 & 2.724 \\
\hline 73 & 1.341 & 1.752 & 2.027 & 2.301 & 2.712 \\
\hline 74 & 1.328 & 1.739 & 2.014 & 2.288 & 2.699 \\
\hline 75 & 1.314 & 1.726 & 2.000 & 2.274 & 2.686 \\
\hline 76 & 1.300 & 1.711 & 1.985 & 2.259 & 2.671 \\
\hline 77 & 1.284 & 1.695 & 1.969 & 2.244 & 2.655 \\
\hline 78 & 1.266 & 1.678 & 1.952 & 2.226 & 2.638 \\
\hline 79 & 1.248 & 1.659 & 1.933 & 2.208 & 2.619 \\
\hline 80 & 1.227 & 1.639 & 1.913 & 2.187 & 2.599 \\
\hline
\end{tabular}


Table IV. I 4

Bone Mineral Density Z score values of arm for female population

Age

-

7

8
Z SCORE

$\begin{array}{lllll}-2.5 & -1.0 & 0.0 & 1.0 & 2.5\end{array}$

$\begin{array}{lllll}0.346 & 0.479 & 0.567 & 0.655 & 0.788\end{array}$

$\begin{array}{lllll}0.375 & 0.508 & 0.596 & 0.685 & 0.817\end{array}$

$\begin{array}{llllll}9 & 0.403 & 0.536 & 0.624 & 0.712 & 0.845\end{array}$

\begin{tabular}{llllll}
\hline 12 & 0.476 & 0.609 & 0.697 & 0.786 & 0.918 \\
\hline
\end{tabular}

$\begin{array}{llllll}13 & 0.498 & 0.630 & 0.718 & 0.807 & 0.939\end{array}$

\begin{tabular}{llllll}
\hline 14 & 0.517 & 0.650 & 0.738 & 0.826 & 0.959 \\
\hline 15 & 0.535 & 0.667 & 0.756 & 0.844 & 0.977
\end{tabular}

\begin{tabular}{llllll}
\hline 16 & 0.551 & 0.684 & 0.772 & 0.860 & 0.993 \\
\hline 17 & 0.566 & 0.698 & 0.787 & 0.875 & 1.007 \\
\hline 18 & 0.578 & 0.711 & 0.799 & 0.888 & 1.020
\end{tabular}

\begin{tabular}{llllll}
\hline 19 & 0.590 & 0.722 & 0.811 & 0.899 & 1.032 \\
\hline 20 & 0.599 & 0.732 & 0.820 & 0.909 & 1.041 \\
\hline 21 & 0.607 & 0.740 & 0.828 & 0.917 & 1.049
\end{tabular}

\begin{tabular}{llllll}
\hline 22 & 0.614 & 0.747 & 0.835 & 0.924 & 1.056
\end{tabular}

\begin{tabular}{llllll}
\hline 23 & 0.620 & 0.752 & 0.841 & 0.929 & 1.062 \\
\hline 24 & 0.624 & 0.757 & 0.845 & 0.933 & 1.066
\end{tabular}

\begin{tabular}{llllll}
\hline 25 & 0.628 & 0.760 & 0.849 & 0.937 & 1.070 \\
\hline 26 & 0.630 & 0.763 & 0.851 & 0.940 & 1.072
\end{tabular}

\begin{tabular}{llllll}
26 & 0.630 & 0.763 & 0.851 & 0.940 & 1.072 \\
\hline 27 & 0.632 & 0.765 & 0.853 & 0.942 & 1.074 \\
\hline 28 & 0.634 & 0.766 & 0.855 & 0.943 & 1.076
\end{tabular}

\begin{tabular}{llllll}
\hline 28 & 0.634 & 0.766 & 0.855 & 0.943 & 1.076 \\
\hline 29 & 0.635 & 0.767 & 0.856 & 0.944 & 1.077 \\
\hline 30 & 0.636 & 0.768 & 0.857 & 0.945 & 1.077 \\
\hline 31 & 0.636 & 0.769 & 0.857 & 0.945 & 1.078 \\
\hline 32 & 0.636 & 0.769 & 0.857 & 0.946 & 1.078 \\
\hline 33 & 0.636 & 0.769 & 0.857 & 0.946 & 1.078 \\
\hline 34 & 0.636 & 0.769 & 0.857 & 0.945 & 1.078 \\
\hline 35 & 0.636 & 0.768 & 0.857 & 0.945 & 1.077 \\
\hline 36 & 0.635 & 0.767 & 0.856 & 0.944 & 1.077 \\
\hline 37 & 0.634 & 0.766 & 0.855 & 0.943 & 1.076 \\
\hline 38 & 0.633 & 0.765 & 0.853 & 0.942 & 1.074 \\
\hline 39 & 0.631 & 0.764 & 0.852 & 0.940 & 1.073 \\
\hline 40 & 0.629 & 0.762 & 0.850 & 0.939 & 1.071 \\
\hline 41 & 0.627 & 0.760 & 0.848 & 0.936 & 1.069 \\
\hline 42 & 0.625 & 0.758 & 0.846 & 0.934 & 1.067 \\
\hline 43 & 0.622 & 0.755 & 0.843 & 0.932 & 1.064 \\
\hline 44 & 0.620 & 0.752 & 0.841 & 0.929 & 1.062
\end{tabular}


Table IV.I5

Bone Mineral Density Z score values of proximal femur for female population

\begin{tabular}{|c|c|c|c|c|c|}
\hline \multirow{2}{*}{ Age } & \multicolumn{5}{|c|}{ Z SCORE } \\
\hline & -2.5 & -1.0 & 0.0 & 1.0 & 2.5 \\
\hline 7 & 0.588 & 0.719 & 0.806 & 0.893 & 1.023 \\
\hline 8 & 0.636 & 0.767 & 0.854 & 0.941 & 1.072 \\
\hline 9 & 0.681 & 0.812 & 0.899 & 0.986 & 1.117 \\
\hline 10 & 0.723 & 0.854 & $0.94 I$ & 1.028 & 1.159 \\
\hline II & 0.762 & 0.893 & 0.980 & 1.067 & 1.197 \\
\hline 12 & 0.797 & 0.928 & 1.015 & 1.102 & 1.233 \\
\hline 13 & 0.829 & 0.960 & 1.047 & 1.134 & 1.265 \\
\hline 14 & 0.858 & 0.989 & 1.076 & 1.163 & 1.294 \\
\hline 15 & 0.884 & 1.014 & 1.101 & 1.188 & 1.319 \\
\hline 16 & 0.906 & 1.037 & I.I24 & 1.211 & $|.34|$ \\
\hline 17 & 0.925 & 1.056 & 1.143 & 1.230 & 1.360 \\
\hline 18 & $0.94 I$ & 1.071 & I.I58 & 1.245 & 1.376 \\
\hline 19 & 0.953 & 1.084 & I.I7| & 1.258 & 1.389 \\
\hline 20 & 0.962 & 1.093 & I. 180 & 1.267 & 1.398 \\
\hline 21 & 0.969 & 1.099 & I.187 & 1.274 & 1.404 \\
\hline 22 & 0.973 & 1.103 & 1.190 & 1.277 & 1.408 \\
\hline 23 & 0.974 & 1.105 & I.192 & 1.279 & 1.410 \\
\hline 24 & 0.974 & 1.105 & 1.192 & 1.279 & 1.409 \\
\hline 25 & 0.972 & 1.103 & 1.190 & 1.277 & 1.408 \\
\hline 26 & 0.969 & 1.100 & I.I87 & 1.274 & 1.405 \\
\hline 27 & 0.966 & 1.096 & 1.183 & I.27I & 1.401 \\
\hline 28 & 0.962 & 1.092 & 1.179 & 1.266 & 1.397 \\
\hline 29 & 0.958 & 1.088 & I.I75 & 1.262 & 1.393 \\
\hline 30 & 0.954 & 1.084 & I.17| & 1.258 & 1.389 \\
\hline 31 & 0.950 & 1.081 & I.I68 & 1.255 & 1.385 \\
\hline 32 & 0.946 & I.077 & I.I64 & 1.251 & 1.382 \\
\hline 33 & 0.943 & 1.074 & 1.161 & 1.248 & 1.379 \\
\hline 34 & 0.940 & I.07| & I.I58 & 1.245 & 1.376 \\
\hline 35 & 0.937 & 1.068 & I.I55 & 1.242 & 1.373 \\
\hline 36 & 0.935 & 1.065 & I.I52 & 1.239 & 1.370 \\
\hline 37 & 0.932 & 1.062 & 1.150 & 1.237 & 1.367 \\
\hline 38 & 0.929 & 1.060 & I.I47 & 1.234 & 1.365 \\
\hline 39 & 0.927 & 1.057 & I.145 & 1.232 & 1.362 \\
\hline 40 & 0.924 & 1.055 & I.I42 & 1.229 & 1.360 \\
\hline 41 & 0.922 & 1.053 & 1.140 & 1.227 & 1.357 \\
\hline 42 & 0.919 & 1.050 & I.I37 & 1.224 & 1.355 \\
\hline 43 & 0.917 & 1.048 & 1.135 & 1.222 & 1.352 \\
\hline 44 & 0.914 & 1.045 & 1.132 & 1.219 & 1.350 \\
\hline
\end{tabular}

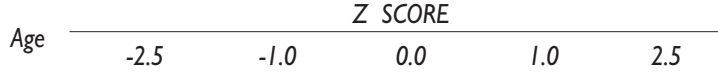

\begin{tabular}{llllll}
45 & 0.912 & 1.042 & 1.129 & 1.217 & 1.347 \\
\hline 46 & 0.909 & 1.040 & 1.127 & 1.214 & 1.344 \\
\hline 47 & 0.906 & 1.037 & 1.124 & 1.211 & 1.342 \\
\hline 48 & 0.903 & 1.034 & 1.121 & 1.208 & 1.339 \\
\hline 49 & 0.900 & 1.031 & 1.118 & 1.205 & 1.335 \\
\hline 50 & 0.897 & 1.027 & 1.114 & 1.201 & 1.332 \\
\hline 51 & 0.893 & 1.024 & 1.111 & 1.198 & 1.328 \\
\hline 52 & 0.889 & 1.020 & 1.107 & 1.194 & 1.324 \\
\hline 53 & 0.885 & 1.016 & 1.103 & 1.190 & 1.320
\end{tabular}

\begin{tabular}{llllll}
\hline 53 & 0.885 & 1.016 & 1.103 & 1.190 & 1.320 \\
\hline 54 & 0.880 & 1.011 & 1.098 & 1.185 & 1.316 \\
\hline 55 & 0.876 & 1.006 & 1.093 & 1.180 & 1.311 \\
\hline 56 & 0.871 & 1.001 & 1.088 & 1.175 & 1.306
\end{tabular}

\begin{tabular}{llllll}
\hline 57 & 0.865 & 0.996 & 1.083 & 1.170 & 1.300 \\
\hline 58 & 0.859 & 0.990 & 1.077 & 1.164 & 1.295 \\
\hline 59 & 0.853 & 0.983 & 1.071 & 1.158 & 1.288
\end{tabular}

\begin{tabular}{llllll}
\hline 60 & 0.846 & 0.977 & 1.064 & 1.151 & 1.281 \\
\hline 61 & 0.839 & 0.969 & 1.057 & 1.144 & 1.274 \\
\hline
\end{tabular}

\begin{tabular}{llllll}
\hline 62 & 0.831 & 0.962 & 1.049 & 1.136 & 1.267 \\
\hline 63 & 0.823 & 0.953 & 1.041 & 1.128 & 1.258 \\
\hline 64 & 0.814 & 0.945 & 1.032 & 1.119 & 1.249 \\
\hline 65 & 0.805 & 0.935 & 1.022 & 1.110 & 1.240 \\
\hline
\end{tabular}

\begin{tabular}{llllll}
\hline 66 & 0.795 & 0.926 & 1.013 & 1.100 & 1.230 \\
\hline 67 & 0.785 & 0.916 & 1.003 & 1.090 & 1.221
\end{tabular}

\begin{tabular}{llllll}
\hline 68 & 0.775 & 0.906 & 0.993 & 1.080 & 1.211 \\
\hline 69 & 0.765 & 0.896 & 0.983 & 1.070 & 1.201
\end{tabular}

\begin{tabular}{llllll}
\hline 70 & 0.756 & 0.887 & 0.974 & 1.061 & 1.191 \\
\hline 71 & 0.747 & 0.878 & 0.965 & 1.052 & 1.182 \\
\hline 72 & 0.739 & 0.869 & 0.956 & 1.043 & 1.174 \\
\hline 73 & 0.731 & 0.862 & 0.949 & 1.036 & 1.167 \\
\hline 74 & 0.725 & 0.855 & 0.943 & 1.030 & 1.160 \\
\hline 75 & 0.720 & 0.850 & 0.937 & 1.024 & 1.155 \\
\hline 76 & 0.716 & 0.846 & 0.934 & 1.021 & 1.151 \\
\hline 77 & 0.714 & 0.844 & 0.931 & 1.018 & 1.149 \\
\hline 78 & 0.711 & 0.842 & 0.929 & 1.016 & 1.147 \\
\hline 79 & 0.709 & 0.840 & 0.927 & 1.014 & 1.145 \\
\hline 80 & 0.707 & 0.838 & 0.925 & 1.012 & 1.143
\end{tabular}


Table IV.16

Bone Mineral Density Z score values of trunk for female population

\begin{tabular}{|c|c|c|c|c|c|}
\hline \multirow{2}{*}{ Age } & \multicolumn{5}{|c|}{ Z SCORE } \\
\hline & -2.5 & -1.0 & 0.0 & 1.0 & 2.5 \\
\hline 7 & 0.446 & 0.565 & 0.644 & 0.724 & 0.843 \\
\hline 8 & 0.483 & 0.602 & 0.682 & 0.761 & 0.880 \\
\hline 9 & 0.518 & 0.638 & 0.717 & 0.797 & 0.916 \\
\hline 10 & 0.551 & $0.67 \mid$ & 0.750 & 0.830 & 0.949 \\
\hline II & 0.582 & 0.701 & 0.781 & 0.860 & 0.980 \\
\hline 12 & 0.611 & 0.730 & 0.809 & 0.889 & 1.008 \\
\hline 13 & 0.637 & 0.756 & 0.836 & 0.915 & 1.034 \\
\hline 14 & 0.661 & 0.780 & 0.860 & 0.939 & 1.058 \\
\hline 15 & 0.683 & 0.802 & 0.881 & 0.961 & 1.080 \\
\hline 16 & 0.702 & 0.821 & 0.901 & 0.980 & 1.099 \\
\hline 17 & 0.719 & 0.838 & 0.918 & 0.997 & 1.117 \\
\hline 18 & 0.734 & 0.853 & 0.933 & 1.012 & 1.132 \\
\hline 19 & 0.747 & 0.866 & 0.946 & 1.025 & 1.144 \\
\hline 20 & 0.757 & 0.876 & 0.956 & 1.035 & 1.155 \\
\hline 21 & 0.766 & 0.885 & 0.964 & 1.044 & 1.163 \\
\hline 22 & 0.772 & 0.891 & 0.971 & 1.050 & 1.170 \\
\hline 23 & 0.777 & 0.896 & 0.976 & 1.055 & 1.174 \\
\hline 24 & $0.78 I$ & 0.900 & 0.979 & 1.059 & 1.178 \\
\hline 25 & 0.783 & 0.902 & 0.981 & 1.061 & 1.180 \\
\hline 26 & 0.784 & 0.903 & 0.983 & 1.062 & 1.181 \\
\hline 27 & 0.784 & 0.904 & 0.983 & 1.063 & 1.182 \\
\hline 28 & 0.784 & 0.903 & 0.983 & 1.062 & 1.182 \\
\hline 29 & 0.784 & 0.903 & 0.982 & 1.062 & 1.181 \\
\hline 30 & 0.783 & 0.902 & 0.982 & 1.061 & 1.180 \\
\hline 31 & 0.782 & 0.901 & 0.981 & 1.060 & 1.179 \\
\hline 32 & $0.78 I$ & 0.900 & 0.980 & 1.059 & 1.178 \\
\hline 33 & 0.780 & 0.899 & 0.978 & 1.058 & 1.177 \\
\hline 34 & 0.778 & 0.897 & 0.977 & 1.056 & 1.175 \\
\hline 35 & 0.776 & 0.896 & 0.975 & 1.055 & 1.174 \\
\hline 36 & 0.775 & 0.894 & 0.973 & 1.053 & 1.172 \\
\hline 37 & 0.773 & 0.892 & 0.971 & 1.051 & 1.170 \\
\hline 38 & $0.77 \mid$ & 0.890 & 0.969 & 1.049 & 1.168 \\
\hline 39 & 0.768 & 0.887 & 0.967 & 1.046 & 1.166 \\
\hline 40 & 0.766 & 0.885 & 0.964 & 1.044 & 1.163 \\
\hline 41 & 0.763 & 0.882 & 0.962 & 1.041 & 1.161 \\
\hline 42 & 0.760 & 0.880 & 0.959 & 1.039 & 1.158 \\
\hline 43 & 0.757 & 0.877 & 0.956 & 1.036 & 1.155 \\
\hline 44 & 0.754 & 0.874 & 0.953 & 1.032 & 1.152 \\
\hline
\end{tabular}

\begin{tabular}{llllll} 
Z Z SCORE \\
\cline { 2 - 5 } & -2.5 & -1.0 & 0.0 & 1.0 & 2.5
\end{tabular}

\begin{tabular}{|c|c|c|c|c|c|}
\hline 45 & $0.75 I$ & 0.870 & 0.950 & 1.029 & I. 148 \\
\hline 46 & 0.748 & 0.867 & 0.946 & 1.026 & 1.145 \\
\hline 47 & 0.744 & 0.863 & 0.943 & 1.022 & 1.141 \\
\hline 48 & 0.740 & 0.860 & 0.939 & 1.019 & 1.138 \\
\hline 49 & 0.737 & 0.856 & 0.935 & 1.015 & 1.134 \\
\hline 50 & 0.733 & 0.852 & 0.931 & 1.011 & 1.130 \\
\hline 51 & 0.728 & 0.848 & 0.927 & 1.007 & 1.126 \\
\hline 52 & 0.724 & 0.843 & 0.923 & 1.002 & 1.121 \\
\hline 53 & 0.720 & 0.839 & 0.918 & 0.998 & 1.117 \\
\hline 54 & 0.715 & 0.834 & 0.914 & 0.993 & 1.112 \\
\hline 55 & 0.710 & 0.830 & 0.909 & 0.989 & 1.108 \\
\hline 56 & 0.706 & 0.825 & 0.904 & 0.984 & 1.103 \\
\hline 57 & 0.701 & 0.820 & 0.899 & 0.979 & 1.098 \\
\hline 58 & 0.695 & 0.815 & 0.894 & 0.974 & 1.093 \\
\hline 59 & 0.690 & 0.809 & 0.889 & 0.968 & 1.088 \\
\hline 60 & 0.685 & 0.804 & 0.884 & 0.963 & 1.082 \\
\hline 61 & 0.679 & 0.799 & 0.878 & 0.957 & 1.077 \\
\hline 62 & 0.674 & 0.793 & 0.872 & 0.952 & I.07I \\
\hline 63 & 0.668 & 0.787 & 0.867 & 0.946 & 1.065 \\
\hline 64 & 0.662 & 0.781 & 0.861 & 0.940 & 1.059 \\
\hline 65 & 0.656 & 0.775 & 0.855 & 0.934 & 1.053 \\
\hline 66 & 0.650 & 0.769 & 0.849 & 0.928 & 1.047 \\
\hline 67 & 0.644 & 0.763 & 0.843 & 0.922 & 1.041 \\
\hline 68 & 0.638 & 0.757 & 0.837 & 0.916 & 1.035 \\
\hline 69 & 0.632 & 0.751 & 0.831 & 0.910 & 1.029 \\
\hline 70 & 0.626 & 0.745 & 0.825 & 0.904 & 1.023 \\
\hline 71 & 0.620 & 0.740 & 0.819 & 0.899 & 1.018 \\
\hline 72 & 0.615 & 0.734 & 0.814 & 0.893 & 1.012 \\
\hline 73 & 0.610 & 0.729 & 0.809 & 0.888 & 1.007 \\
\hline 74 & 0.605 & 0.724 & 0.804 & 0.883 & 1.002 \\
\hline 75 & 0.601 & 0.720 & 0.799 & 0.879 & 0.998 \\
\hline 76 & 0.597 & 0.716 & 0.795 & 0.875 & 0.994 \\
\hline 77 & 0.593 & 0.712 & 0.792 & 0.871 & 0.991 \\
\hline 78 & 0.590 & 0.710 & 0.789 & 0.868 & 0.988 \\
\hline 79 & 0.588 & 0.707 & 0.787 & 0.866 & 0.985 \\
\hline 80 & 0.586 & 0.706 & 0.785 & 0.865 & 0.984 \\
\hline
\end{tabular}


Table IV.I7

Bone Mineral Density Z score values of ribs for female population

\begin{tabular}{|c|c|c|c|c|c|}
\hline \multirow{2}{*}{ Age } & \multicolumn{5}{|c|}{ Z SCORE } \\
\hline & -2.5 & -1.0 & 0.0 & 1.0 & 2.5 \\
\hline 7 & 0.400 & 0.492 & 0.553 & 0.614 & 0.706 \\
\hline 8 & 0.423 & 0.515 & 0.576 & 0.637 & 0.729 \\
\hline 9 & 0.444 & 0.536 & 0.598 & 0.659 & $0.75 I$ \\
\hline 10 & 0.465 & 0.556 & 0.618 & 0.679 & 0.771 \\
\hline II & 0.483 & 0.575 & 0.636 & 0.698 & 0.790 \\
\hline 12 & 0.500 & 0.592 & 0.654 & 0.715 & 0.807 \\
\hline 13 & 0.516 & 0.608 & 0.669 & 0.730 & 0.822 \\
\hline 14 & 0.530 & 0.622 & 0.683 & 0.745 & 0.837 \\
\hline 15 & 0.543 & 0.635 & 0.696 & 0.757 & 0.849 \\
\hline 16 & 0.554 & 0.646 & 0.707 & 0.768 & 0.860 \\
\hline 17 & 0.564 & 0.656 & 0.717 & 0.778 & 0.870 \\
\hline 18 & 0.572 & 0.664 & 0.725 & 0.786 & 0.878 \\
\hline 19 & 0.578 & 0.670 & 0.732 & 0.793 & 0.885 \\
\hline 20 & 0.584 & 0.676 & 0.737 & 0.798 & 0.890 \\
\hline 21 & 0.588 & 0.680 & $0.74 I$ & 0.802 & 0.894 \\
\hline 22 & 0.590 & 0.682 & 0.744 & 0.805 & 0.897 \\
\hline 23 & 0.592 & 0.684 & 0.745 & 0.806 & 0.898 \\
\hline 24 & 0.593 & 0.685 & 0.746 & 0.807 & 0.899 \\
\hline 25 & 0.593 & 0.685 & 0.746 & 0.807 & 0.899 \\
\hline 26 & 0.592 & 0.684 & 0.745 & 0.807 & 0.899 \\
\hline 27 & 0.591 & 0.683 & 0.744 & 0.806 & 0.898 \\
\hline 28 & 0.590 & 0.682 & 0.743 & 0.804 & 0.896 \\
\hline 29 & 0.589 & 0.681 & 0.742 & 0.803 & 0.895 \\
\hline 30 & 0.587 & 0.679 & 0.740 & 0.802 & 0.894 \\
\hline 31 & 0.586 & 0.678 & 0.739 & 0.800 & 0.892 \\
\hline 32 & 0.584 & 0.676 & 0.738 & 0.799 & 0.891 \\
\hline 33 & 0.583 & 0.675 & 0.736 & 0.798 & 0.889 \\
\hline 34 & 0.582 & 0.674 & 0.735 & 0.796 & 0.888 \\
\hline 35 & 0.580 & 0.672 & 0.734 & 0.795 & 0.887 \\
\hline 36 & 0.579 & $0.67 \mid$ & 0.732 & 0.793 & 0.885 \\
\hline 37 & 0.578 & 0.669 & 0.731 & 0.792 & 0.884 \\
\hline 38 & 0.576 & 0.668 & 0.729 & $0.79 \mid$ & 0.883 \\
\hline 39 & 0.575 & 0.667 & 0.728 & 0.789 & 0.881 \\
\hline 40 & 0.573 & 0.665 & 0.726 & 0.788 & 0.880 \\
\hline 41 & 0.572 & 0.664 & 0.725 & 0.786 & 0.878 \\
\hline 42 & 0.570 & 0.662 & 0.723 & 0.785 & 0.877 \\
\hline 43 & 0.568 & 0.660 & 0.722 & 0.783 & 0.875 \\
\hline 44 & 0.567 & 0.659 & 0.720 & $0.78 I$ & 0.873 \\
\hline
\end{tabular}

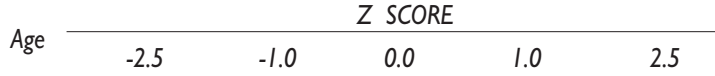

\begin{tabular}{llllll}
45 & 0.565 & 0.657 & 0.718 & 0.780 & 0.872 \\
\hline 46 & 0.563 & 0.655 & 0.717 & 0.778 & 0.870 \\
\hline 47 & 0.562 & 0.653 & 0.715 & 0.776 & 0.868 \\
\hline 48 & 0.560 & 0.652 & 0.713 & 0.774 & 0.866 \\
\hline 49 & 0.558 & 0.650 & 0.711 & 0.772 & 0.864 \\
\hline
\end{tabular}

\begin{tabular}{rlllll}
\hline 50 & 0.556 & 0.648 & 0.709 & 0.770 & 0.862 \\
\hline 51 & 0.553 & 0.645 & 0.707 & 0.768 & 0.860 \\
\hline 52 & 0.551 & 0.643 & 0.704 & 0.766 & 0.858 \\
\hline 53 & 0.549 & 0.641 & 0.702 & 0.763 & 0.855
\end{tabular}

\begin{tabular}{llllll}
\hline 53 & 0.549 & 0.641 & 0.702 & 0.763 & 0.855 \\
\hline 54 & 0.546 & 0.638 & 0.700 & 0.761 & 0.853 \\
\hline 55 & 0.544 & 0.636 & 0.697 & 0.758 & 0.850
\end{tabular}

\begin{tabular}{rlllll}
\hline 56 & 0.541 & 0.633 & 0.694 & 0.756 & 0.848 \\
\hline 57 & 0.538 & 0.630 & 0.692 & 0.753 & 0.845 \\
\hline 58 & 0.536 & 0.627 & 0.689 & 0.750 & 0.842 \\
\hline 59 & 0.532 & 0.624 & 0.686 & 0.747 & 0.839 \\
\hline
\end{tabular}

\begin{tabular}{llllll}
\hline 60 & 0.529 & 0.621 & 0.683 & 0.744 & 0.836 \\
\hline 61 & 0.526 & 0.618 & 0.679 & 0.741 & 0.832 \\
\hline 62 & 0.523 & 0.615 & 0.676 & 0.737 & 0.829 \\
\hline 63 & 0.519 & 0.611 & 0.672 & 0.733 & 0.825
\end{tabular}

\begin{tabular}{llllll}
63 & 0.519 & 0.611 & 0.672 & 0.733 & 0.825 \\
\hline 64 & 0.515 & 0.607 & 0.668 & 0.730 & 0.822 \\
\hline 65 & 0.511 & 0.603 & 0.665 & 0.726 & 0.818 \\
\hline 66 & 0.507 & 0.599 & 0.661 & 0.722 & 0.814
\end{tabular}

\begin{tabular}{llllll}
66 & 0.507 & 0.599 & 0.661 & 0.722 & 0.814 \\
\hline 67 & 0.503 & 0.595 & 0.656 & 0.718 & 0.810 \\
\hline 68 & 0.499 & 0.591 & 0.652 & 0.713 & 0.805 \\
\hline 69 & 0.494 & 0.586 & 0.648 & 0.709 & 0.801 \\
\hline 70 & 0.490 & 0.582 & 0.643 & 0.705 & 0.796 \\
\hline 71 & 0.485 & 0.577 & 0.639 & 0.700 & 0.792 \\
\hline 72 & 0.481 & 0.573 & 0.634 & 0.695 & 0.787 \\
\hline 73 & 0.476 & 0.568 & 0.629 & 0.691 & 0.783 \\
\hline 74 & 0.471 & 0.563 & 0.625 & 0.686 & 0.778 \\
\hline 75 & 0.467 & 0.559 & 0.620 & 0.681 & 0.773 \\
\hline 76 & 0.462 & 0.554 & 0.615 & 0.677 & 0.769 \\
\hline 77 & 0.457 & 0.549 & 0.611 & 0.672 & 0.764 \\
\hline 78 & 0.453 & 0.545 & 0.606 & 0.667 & 0.759 \\
\hline 79 & 0.448 & 0.540 & 0.601 & 0.663 & 0.755 \\
\hline 80 & 0.443 & 0.535 & 0.597 & 0.658 & 0.750
\end{tabular}


Table IV.18

Bone Mineral Density Z score values of pelvis for female population

Age 8

\begin{tabular}{|c|c|c|c|c|c|}
\hline 7 & 0.476 & 0.636 & 0.742 & 0.849 & 1.009 \\
\hline 8 & 0.523 & 0.683 & 0.790 & 0.897 & 1.057 \\
\hline 9 & 0.568 & 0.728 & 0.835 & 0.942 & 1.102 \\
\hline 10 & 0.610 & 0.770 & 0.877 & 0.983 & 1.144 \\
\hline II & 0.649 & 0.809 & 0.916 & 1.022 & 1.182 \\
\hline 12 & 0.685 & 0.845 & 0.952 & 1.058 & 1.218 \\
\hline 13 & 0.718 & 0.878 & 0.985 & 1.091 & $\mid .251$ \\
\hline 14 & 0.748 & 0.908 & 1.015 & 1.121 & 1.281 \\
\hline 15 & 0.775 & 0.935 & 1.042 & 1.148 & 1.309 \\
\hline 16 & 0.799 & 0.959 & 1.066 & 1.173 & 1.333 \\
\hline 17 & 0.820 & 0.980 & 1.087 & 1.194 & 1.354 \\
\hline 18 & 0.839 & 0.999 & 1.105 & 1.212 & 1.372 \\
\hline 19 & 0.854 & 1.014 & 1.121 & 1.227 & 1.388 \\
\hline 20 & 0.866 & 1.026 & 1.133 & 1.240 & 1.400 \\
\hline 21 & 0.876 & 1.036 & 1.143 & 1.250 & 1.410 \\
\hline 22 & 0.883 & 1.044 & 1.150 & 1.257 & 1.417 \\
\hline 23 & 0.889 & 1.049 & I.I56 & 1.262 & 1.422 \\
\hline 24 & 0.892 & 1.052 & 1.159 & 1.266 & 1.426 \\
\hline 25 & 0.894 & 1.054 & 1.161 & 1.268 & 1.428 \\
\hline 26 & 0.895 & 1.055 & 1.162 & 1.268 & 1.429 \\
\hline 27 & 0.895 & 1.055 & 1.161 & 1.268 & 1.428 \\
\hline 28 & 0.894 & 1.054 & 1.161 & 1.267 & 1.427 \\
\hline 29 & 0.892 & 1.052 & I.159 & 1.266 & 1.426 \\
\hline 30 & 0.891 & I.05I & 1.158 & 1.265 & 1.425 \\
\hline 31 & 0.889 & 1.049 & 1.156 & 1.263 & 1.423 \\
\hline 32 & 0.888 & 1.048 & 1.155 & 1.261 & $\mid .421$ \\
\hline 33 & 0.886 & 1.046 & 1.153 & 1.259 & 1.420 \\
\hline 34 & 0.884 & 1.044 & 1.151 & 1.257 & 1.418 \\
\hline 35 & 0.882 & 1.042 & 1.149 & 1.255 & 1.416 \\
\hline 36 & 0.880 & 1.040 & 1.146 & 1.253 & 1.413 \\
\hline 37 & 0.877 & 1.037 & I.I44 & 1.251 & $1.4 \mathrm{II}$ \\
\hline 38 & 0.875 & 1.035 & 1.142 & 1.248 & 1.409 \\
\hline 39 & 0.872 & 1.032 & 1.139 & 1.246 & 1.406 \\
\hline 40 & 0.869 & 1.029 & 1.136 & 1.243 & 1.403 \\
\hline $4 I$ & 0.866 & 1.027 & 1.133 & 1.240 & 1.400 \\
\hline 42 & 0.863 & 1.023 & 1.130 & 1.237 & 1.397 \\
\hline 43 & 0.860 & 1.020 & 1.127 & 1.234 & 1.394 \\
\hline 44 & 0.857 & 1.017 & 1.123 & 1.230 & 1.390 \\
\hline
\end{tabular}

\begin{tabular}{ccccc} 
Z Z SCORE \\
\cline { 2 - 6 }
\end{tabular}

\begin{tabular}{|c|c|c|c|c|c|}
\hline 45 & 0.853 & 1.013 & 1.120 & 1.226 & 1.387 \\
\hline 46 & 0.849 & 1.009 & 1.116 & 1.223 & 1.383 \\
\hline 47 & 0.845 & 1.005 & 1.112 & 1.219 & 1.379 \\
\hline 48 & $0.84 I$ & 1.001 & 1.108 & 1.214 & 1.374 \\
\hline 49 & 0.836 & 0.996 & 1.103 & 1.210 & 1.370 \\
\hline 50 & 0.832 & 0.992 & 1.099 & 1.205 & 1.365 \\
\hline 51 & 0.827 & 0.987 & 1.094 & 1.201 & 1.361 \\
\hline 52 & 0.822 & 0.982 & 1.089 & 1.195 & 1.356 \\
\hline 53 & 0.817 & 0.977 & 1.083 & 1.190 & 1.350 \\
\hline 54 & 0.811 & 0.971 & 1.078 & 1.185 & 1.345 \\
\hline 55 & 0.805 & 0.965 & 1.072 & 1.179 & 1.339 \\
\hline 56 & 0.799 & 0.960 & 1.066 & 1.173 & 1.333 \\
\hline 57 & 0.793 & 0.953 & 1.060 & 1.167 & 1.327 \\
\hline 58 & 0.787 & 0.947 & 1.054 & 1.160 & 1.320 \\
\hline 59 & 0.780 & 0.940 & 1.047 & I.I54 & 1.314 \\
\hline 60 & 0.773 & 0.933 & 1.040 & 1.147 & 1.307 \\
\hline 61 & 0.766 & 0.926 & 1.033 & 1.140 & 1.300 \\
\hline 62 & 0.758 & 0.919 & 1.025 & 1.132 & 1.292 \\
\hline 63 & 0.751 & 0.911 & 1.018 & 1.124 & 1.284 \\
\hline 64 & 0.743 & 0.903 & 1.010 & 1.116 & 1.276 \\
\hline 65 & 0.734 & 0.895 & 1.001 & 1.108 & 1.268 \\
\hline 66 & 0.726 & 0.886 & 0.993 & 1.100 & 1.260 \\
\hline 67 & 0.717 & 0.878 & 0.984 & 1.091 & $1.25 \mid$ \\
\hline 68 & 0.709 & 0.869 & 0.976 & 1.082 & 1.243 \\
\hline 69 & 0.700 & 0.861 & 0.967 & 1.074 & 1.234 \\
\hline 70 & 0.692 & 0.852 & 0.959 & 1.066 & 1.226 \\
\hline 71 & 0.684 & 0.844 & 0.951 & 1.058 & 1.218 \\
\hline 72 & 0.677 & 0.837 & 0.944 & 1.050 & 1.210 \\
\hline 73 & 0.670 & 0.830 & 0.937 & 1.043 & 1.203 \\
\hline 74 & 0.663 & 0.823 & 0.930 & 1.037 & 1.197 \\
\hline 75 & 0.658 & 0.818 & 0.924 & 1.031 & 1.191 \\
\hline 76 & 0.653 & 0.813 & 0.919 & 1.026 & 1.186 \\
\hline 77 & 0.649 & 0.809 & 0.915 & 1.022 & 1.182 \\
\hline 78 & 0.645 & 0.806 & 0.912 & 1.019 & 1.179 \\
\hline 79 & 0.643 & 0.803 & 0.910 & 1.017 & 1.177 \\
\hline 80 & 0.641 & 0.801 & 0.908 & 1.015 & 1.175 \\
\hline
\end{tabular}




\section{References}

I. Zingmond DS, Melton III LI, Silverman SL. Increasing hip fracture incidence in California Hispanics; 1983 to 2000. Osteoporos Int 2004;15:603-610.

2. Chang KP, Center JR, Nguyen TV, Eisman JA. Incidence of hip and other osteoporotic fractures in elderly men and women: Dubbo osteoporosis epidemiology study. Journal Bone and Min Res 2004;19.

3. Eisman J, Clapham S, Kehoe L. Osteoporosis prevalence and levels of treatment in primary care: the australian bone care study.J Bone Miner Res 2004;19:1969-1975.

4. Siris ES, Brenneman SK, Miller PD, Barrett-Connor E, Chen YT, Sherwood LM, et al. Predictive value of low BMD for I-year fracture outcomes is similar for postmenopausal women ages 50-64 and 65 and older: results from the National Osteoporosis Risk Assessment (NORA).J Bone Miner Res 2004;19:1215-1220.

5. Barrett-Connor E, Siris ES, Wehren LE, Miller PD, Abbott TA, Berger ML, et al. Osteoporosis and fracture risk in women of different ethnic groups.J Bone Miner Res 2005;20:185-194.

6. Nevitt MC, Cummings SR, Stone KL, Palermo L, Black DM, Bauer DC, et al. Risk factors for a first-incident radiographic vertebral fracture in women $>65$ Years of Age: the study of osteoporotic fractures. J Bone Miner Res 2005;20:13|-|40

7. Velasco-Murillo V. Fracturas en mujeres posmenopáusicas en el IMSS: frecuencia y costos de su atención hospitalaria. Gac Méd Méx. 2003; 139:453-458.

8. Morales Torres J, Hernández Ochoa C, Hernández Paz R, Hernández Morales $\mathrm{G}$. Fracturas de cadera en egresos de hospitales de León, Gto. un indicador econométrico de osteoporosis. Rev Mex Reumat. 1997; I2: I |9-124.

9. Gómez García F. Morbimortalidad de fracturas de cadera en el hospital de traumatología magdalena de las salinas, IMSS. Rev Mex Ortop Traum 1998;2:48-52.

10. Clark P, de la Peña F, Gomez-Garcia F, Orozco JA,Tugwell P. Risk factors for osteoporotic hip fractures in mexicans. Arch Med Res 1998;29:253-257.

I I. Espino DV, Palmer RF, Miles TP et al. Prevalence, incidence, and risk factors associated with hip fractures in community-dwelling older Mexican Americans: results of the Hispanic EPESE study. Establish Population for the Epidemiologic Study for the Elderly. J Am Geriatr Soc 2000;48: I252-I26.

12. Clark P, Lavielle P, Salmerón J Y, Cummings S. Tasas de incidencia de fracturas de cadera en población mexicana mayor de 50 años (abstract). Rev Metab Oseo Min. 2003;I:4I.

13. Clark P, Delezé M, Cons-Molina F, Salmerón J, Palermo L, Cummings SR, et al. Prevalencia de fracturas vertebrales en población Mexicana (abstract). Rev Metab Oseo Min. 2003; I:4I.

14. Kanis JA for the WHO Study Group (1994) Assessment of fracture risk and its application to screening for postmenopausal osteoporosis: Synopsis of a WHO report. Osteoporos Int 4:368-38I.

15.-The Writing Group for the ISCD Position Development Conference. Nomenclature and Decimal Places in Bone Densitometry (2004) JClin Densit, 7: 45-49.

16. Leib ES, Lewiecki EM, Binkley N, Hamdy RC. Official Positions of the International Society for Clinical Densitometry. JClin Densit. 2004;7: I-5.

17. Melton III LJ, Chrischilles EA, Cooper C, Lane AW, Riggs BL. How Many Women Have Osteoporosis?.J Bone Min Res. 1992;7:1005-I0I0.
18. Arzac P, Tamayo J. How many women have osteoporosis in medica sur osteoporosis clinic.J Bone Miner Res. 1996; I I:S36I.

19. Melton III LJ, BeckTJ,Amin S, Khosla S,Achenbach SJ, Oberg AL, Riggs BL. Contributions of bone density and structure to fracture risk assessment in men and women. Osteoporos Int. 2005;16:460-467.

20. Heaney RP,Abrams S, Dawson-Hughes B, Looker A, Marcus R, Matkovic V,Weaver C. Peak bone mass. Osteoporosis Int. 2000; I I:985- 1009.

2I. Chevalley T, Rizzoli R, Hans D, Ferrari S, Bonjour JP. Interaction between calcium intake and menarcheal age on bone mass gain: an eight-year follow-up study from prepuberty to postmenarche.J Clin Endocrinol Metab. 2005;90:44-5I.

22. Richy F,Ethgen O, Bruyere O, MawetA, Reginster JY.Primary prevention of osteoporosis: mass screening scenario or prescreening with questionnaires? an economic perspective.J Bone Min Res. 2004; 19:1955-1960.

23. Kanis JA., Johnell O. Requirements for DXA for the management of osteoporosis in Europe Osteoporos Int. 2005; 16:229-238.

24. Gourlay ML, Miller WC, Richy F, Garrett JM, Hanson LC, Reginster JY. Performance of osteoporosis risk assessment tools in postmenopausal women aged 45-64 years. Osteoporos Int. 2005; 16:921-927.

25. Cummings,S.R. Bone Density Screening:A New Level of Evidence?.Ann Intern Med. 2005;142:217-219.

26. Raisz L. Screening for Osteoporosis. New Eng J Med. 2005;353:164-I7I. 27. Owner's Manual, LUNAR Corp.

28. Lazcano-Ponce E, Tamayo J, Cruz-Valdez A, Díaz R, Hernández B, Del Cueto R, Hernández-Avila M. Peak bone mineral area density and determinants among females aged 9 to 24 years in Mexico. Osteoporos Int. 2003; I4: 539-547.

29. Delezé M, Cons-Molina F, Villa AR, Morales-Torres J, et al. Geographic Differences in Bone Mineral Density of Mexican Women. Osteoporos Int. 2000; I I:562-569.

30. Morales-Torres J, Gutiérrez-Ureña S.The burden of osteoporosis in Latin America. Osteoporos Int. 2004;15:625-632.

31.Johnell O,Kanis JA.An estimate of the worldwide prevalence, mortality and disability associated with hip fracture. Osteoporos Int. 2004;15:897-902.

32. Melton III LJ, Johnell O, Lau E, Mautalen CA, Seeman E. Osteoporosis and the Global Competition for Health Care Resources.J Bone Miner Res. 2004;19:1055-1058.

33. Binkley N, Kiebzak GM, Lewiecki EM, Krueger D, Gangnon RE, Miller PD, et al. Recalculation of the NHANES database SD improves T-score agreement and reduces osteoporosis prevalence.J Bone Miner Res. 2005;20:195-20I. 34.Api M. Letter to the Editor,AreWe TreatingWomen with Postmenopausal Osteoporosis for their low BMD or high fracture risk? J Bone Miner Res. 2005:20:1480-|48|.

35. Hui SL,Slemenda CW, Johnston Jr CC, et al.Age and bone mass as predictors of fracture in a prospective study.J Clin Invest. 1988;81:1804- 809.

36. Lindsay R, Pack S, Li Z. Longitudinal progression of fracture prevalence through a population of postmenopausal women with osteoporosis. Osteoporos Int. 2005; 16:306-3/2.

37. Porias H, Morales Torres J, et al. Consenso Mexicano de Osteoporosis. Rev Metab Oseo Min. 2003; I: I-24.

38. Reza Albarran AA,Vergara LópezA, Mendoza ZubietaV,Martínez Sobaja C, Zúñiga González S, Porias Cuellar HL,Arechavaleta Granell R. Osteoporosis, Posición de la Sociedad Mexicana de Nutrición y Endocrinología. Rev Endoc y Nutr. 2004;12:123-163. 


\section{Appendix}

Table A.I

Comparison for L2-L4 between the Deleze, Cons et al. Classification using the manufacturer's reference values and our Mexican database using T-scores calculated With data for ages 20-39, 30-40 or PBM

\begin{tabular}{|c|c|c|c|c|c|c|}
\hline \multirow[b]{3}{*}{ Groups according to Deleze, Cons et al. } & \multirow[b]{3}{*}{ Normal } & \multirow[b]{3}{*}{ Count } & \multicolumn{3}{|c|}{ Groups according to Mexican data base } & \multirow[t]{2}{*}{ Total } \\
\hline & & & Normal & Osteopenia & Osteoporosis & \\
\hline & & & 1930 & 163 & 0 & 2093 \\
\hline & & $\%$ of total & $45.1 \%$ & $3.8 \%$ & $0.0 \%$ & $48.9 \%$ \\
\hline & Osteopenia & Count & 103 & $|36|$ & 152 & 1616 \\
\hline & & $\%$ of total & $2.4 \%$ & $31.8 \%$ & $3.6 \%$ & $37.8 \%$ \\
\hline & Osteoporosis & Count & 0 & 86 & 484 & 570 \\
\hline & & $\%$ of total & $0.0 \%$ & $2.0 \%$ & $11.3 \%$ & $13.3 \%$ \\
\hline \multirow[t]{2}{*}{ Total } & & Count & 2033 & 1610 & 636 & 4279 \\
\hline & & $\%$ of total & $47.5 \%$ & $37.6 \%$ & $14.9 \%$ & $100.0 \%$ \\
\hline
\end{tabular}

Table A.2

StATISTICAL ANALYSIS FOR L2-L4

\begin{tabular}{lccccl} 
& $\begin{array}{c}\text { Percentage } \\
\text { Deleze Cons et al }\end{array}$ & Mexican & Percentage of differences & Exact probability & Significance of differences \\
Normal & $48.9 \%$ & $47.5 \%$ & 1.2981 & 0.0971 & Not significant \\
\hline Osteopenia & $37.8 \%$ & $37.6 \%$ & 0.1338 & 0.4468 & Not significant \\
\hline Osteoporosis & $13.3 \%$ & $14.9 \%$ & -2.0510 & 0.0201 & Significant \\
\hline
\end{tabular}

Table A.3

Comparison of Classification of femoral neck density between the Deleze, Cons et al. CLAssification using the manufacturer's Reference values and our Mexican database

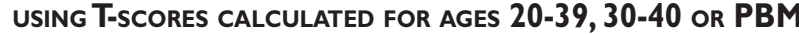

\begin{tabular}{|c|c|c|c|c|c|c|}
\hline \multirow[b]{3}{*}{ Groups according to Deleze Cons et.al. } & \multirow[b]{3}{*}{ Normal } & \multirow[b]{3}{*}{ Count } & \multicolumn{3}{|c|}{ Groups according to Mexican data base } & \multirow{3}{*}{$\begin{array}{c}\text { Total } \\
\text { Normal } \\
2251\end{array}$} \\
\hline & & & Normal & Osteopenia & Osteoporosis & \\
\hline & & & 1600 & 651 & 0 & \\
\hline & & $\%$ of total & $36.8 \%$ & $15.0 \%$ & $.0 \%$ & $51.8 \%$ \\
\hline & Osteopenia & Count & 0 & 428 & 326 & I 754 \\
\hline & & $\%$ of total & $.0 \%$ & $32.8 \%$ & $7.5 \%$ & $40.3 \%$ \\
\hline & Osteoporosis & Count & 0 & 0 & 343 & 343 \\
\hline & & $\%$ of total & $.0 \%$ & $.0 \%$ & $7.9 \%$ & $7.9 \%$ \\
\hline Total & & Count & 1600 & 2079 & 669 & 4348 \\
\hline & & $\%$ of total & $36.8 \%$ & $47.8 \%$ & $15.4 \%$ & $100.0 \%$ \\
\hline
\end{tabular}

Table A.4

StATISTICAL ANALYSIS FOR FEMORAL NECK

\begin{tabular}{|c|c|c|c|c|c|}
\hline \multirow[b]{2}{*}{ Group } & \multicolumn{2}{|c|}{ Percentage } & \multirow[t]{2}{*}{ Percentage of differences } & \multirow{2}{*}{ Exact probability } & \multirow{2}{*}{ Significance of differences } \\
\hline & Deleze Cons et al & Mexican & & & \\
\hline Normal & 51.8 & 36.8 & 14.1218 & 0.0000 & Significant \\
\hline Osteopenia & 40.3 & 47.8 & -7.0043 & 0.0000 & Significant \\
\hline Osteoporosis & 7.9 & 15.4 & -10.8836 & 0.0000 & Significant \\
\hline
\end{tabular}

